\title{
Nonlocal gravity with a Weyl-square term
}

\author{
Giulia Cusin, ${ }^{1}$ Stefano Foffa, ${ }^{1}$ Michele Maggiore, ${ }^{1}$ and Michele Mancarella ${ }^{2,3,4}$ \\ ${ }^{1}$ Département de Physique Théorique and Center for Astroparticle Physics, Université de Genève, \\ 24 quai Ansermet, $\mathrm{CH}-1211$ Genève 4, Switzerland \\ ${ }^{2}$ Institut de physique théorique, Université Paris Saclay CEA, CNRS, 91191 Gif-sur-Yvette, France \\ ${ }^{3}$ Université Paris Sud, 15 rue George Clémenceau, 91405, Orsay, France \\ ${ }^{4}$ Physics Department, Theory Unit, CERN, CH-1211 Genève 23, Switzerland
}

(Received 20 December 2015; published 8 February 2016)

\begin{abstract}
Recent work has shown that modifications of general relativity based on the addition to the action of a nonlocal term $R \square^{-2} R$, or on the addition to the equations of motion of a term involving $\left(g_{\mu \nu} \square^{-1} R\right)$, produce dynamical models of dark energy which are cosmologically viable both at the background level and at the level of cosmological perturbations. We explore a more general class of models based on the addition to the action of terms proportional to $R_{\mu \nu} \square^{-2} R^{\mu \nu}$ and $C_{\mu \nu \rho \sigma} \square^{-2} C^{\mu \nu \rho \sigma}$, where $C_{\mu \nu \rho \sigma}$ is the Weyl tensor. We find that the term $R_{\mu \nu} \square^{-2} R^{\mu \nu}$ does not give a viable background evolution. The nonlocal Weylsquare term, in contrast, does not contribute to the background evolution but we find that, at the level of cosmological perturbations, it gives instabilities in the tensor sector. Thus, only nonlocal terms which depend just on the Ricci scalar $R$ appear to be cosmologically viable. We discuss how these results can provide a hint for the mechanism that might generate these effective nonlocal terms from a fundamental local theory.
\end{abstract}

DOI: 10.1103/PhysRevD.93.043006

\section{INTRODUCTION}

Locality is one of the fundamental principles of quantum field theory. Nevertheless, even in a fundamental local theory, nonlocalities in general appear at an effective level. This can already happen classically, when one integrates out some degree of freedom and obtains an effective theory for the remaining degrees of freedom. Cosmological perturbation theory provides a simple example. In this case, integrating out the short-wavelength modes, one finds effective nonlocal equations for the long-wavelength modes [1]. At the quantum level, nonlocal terms emerge when one computes the effective action that takes into account loops of massless or light particles.

Nonlocal terms featuring inverse powers of the d'Alembertian operator are relevant in the infrared (IR), and it is natural to ask whether such nonlocalities could play a role as IR modifications of general relativity (GR), and in particular whether they could provide a dynamical explanation for dark energy. An attempt in this direction has been done in Refs. [2-4], adding to the Ricci scalar $R$ in the Einstein-Hilbert action a term of the form $R f\left(\square^{-1} R\right)$. In models of this form there is no explicit mass scale, since the function $f$ is dimensionless. However, this class of models has been ruled out by the comparison with cosmological observations [5]. Indeed, after fixing the function $f\left(\square^{-1} R\right)$

Published by the American Physical Society under the terms of the Creative Commons Attribution 3.0 License. Further distribution of this work must maintain attribution to the author(s) and the published article's title, journal citation, and DOI. so that it gives a viable cosmological evolution at the background level, the corresponding cosmological perturbations fail to reproduce structure-formation data.

The situation is more promising for models where the nonlocal terms depend on an explicit mass scale (whose physical origin could be due to mechanisms such as those discussed recently in Ref. [6]). Models of this type have been explored in the context of the degravitation mechanism [7-9]. Partly inspired by these works, as well as by attempts at writing massive gravity in a nonlocal form $[10,11]$, in Ref. [12] a phenomenological modification of gravity was proposed, based on the nonlocal equation of motion

$$
G_{\mu \nu}-(1 / 3) m^{2}\left(g_{\mu \nu} \square^{-1} R\right)^{\mathrm{T}}=8 \pi G T_{\mu \nu},
$$

where the superscript $\mathrm{T}$ denotes the operation of taking the transverse part of a tensor (which is itself a nonlocal operation), and $m^{2}$ is a mass parameter. The extraction of the transverse part ensures that energy-momentum conservation is automatically satisfied. A closed form for the action corresponding to Eq. (1.1) is not known. A related nonlocal model, proposed in Ref. [13], is defined by the action

$$
S=\frac{m_{\mathrm{Pl}}^{2}}{2} \int d^{4} x \sqrt{-g}\left[R-\frac{1}{6} m^{2} R \frac{1}{\square^{2}} R\right],
$$

where $m_{\mathrm{Pl}}=1 / \sqrt{8 \pi G}$ is the reduced Planck mass, and $m$ is a mass parameter. These two models are related by the fact that, linearizing over flat space, they give the same 
equations of motion. However at the full nonlinear level, or linearizing over a background different from Minkowski [such as Friedmann-Robertson-Walker (FRW)], they are different. Both in Eq. (1.1) and in the equations of motion derived from Eq. (1.2), the inverse d'Alembertian is taken to be the one defined using the retarded Green's function, to ensure causality. A nonlocal action such as Eq. (1.2) must indeed be understood as an effective action which takes into account quantum loop effects. For the in-out expectation values of quantum fields the variation of such a nonlocal effective action gives nonlocal equations of motion which depend on the Feynman propagator. However, the equations of motion for the in-in expectation values, which can be obtained with the Schwinger-Keldysh formalism, depend on the inverse d'Alembertian defined with the retarded Green's function, and are therefore causal.

As discussed in Refs. [12-16], in the comparison with cosmological observations the models (1.1) and (1.2) perform remarkably well. They dynamically generate a dark energy and have a realistic background FRW evolution. Their cosmological perturbations are well behaved and their quantitative effects are consistent with cosmic microwave background (CMB), supernovae ( $\mathrm{SNe}$ ), baryon acoustic oscillation (BAO) and structure formation data [15-17]. Implementing the cosmological perturbations of the nonlocal models into a Boltzmann code and performing parameter estimation and a fit to $\mathrm{CMB}$, supernovae and BAO data, one finds that these models fit the data very well, with a $\chi^{2}$ comparable to that of $\Lambda \mathrm{CDM}[18] .{ }^{1}$ It should be stressed that both the model (1.1) and the model (1.2) have the same number of parameters as $\Lambda \mathrm{CDM}$, with the mass $m$ replacing the cosmological constant, and in this sense they are the only existing models that can compete quantitatively with $\Lambda \mathrm{CDM}$ from the point of view of fitting the data, without being merely an extension of $\Lambda \mathrm{CDM}$ with extra free parameters. Further work on these models has been presented in Refs. [20-28].

The purpose of this paper is to investigate whether some possible generalizations of these nonlocal theories are cosmologically viable. We are not driven here by the aim of improving the agreement with the cosmological observations. From this point of view the one-parameter models (1.1) and (1.2) already work so well that, with present data, there is not much point in enlarging the parameter space. Rather, our question is of a more conceptual nature. Indeed, the models (1.1) and (1.2) have been proposed on a purely phenomenological ground. The next step should be to understand how they could be generated from a fundamental local theory. From this point of view, it

\footnotetext{
${ }^{1}$ Actually in Ref. [19], updating the analysis of Ref. [18] using the 2015 Planck data as well as extended BAO data sets, and performing a Bayesian model comparison, it will be shown that the model (1.1) and $\Lambda \mathrm{CDM}$ both fit the data equally well, while the model (1.2), even if by itself fits the data well, is disfavored in the Bayesian comparison with $\Lambda \mathrm{CDM}$ or with the model (1.1).
}

is important to understand whether the fundamental local theory should generate exactly a nonlocal structure such as that given in Eq. (1.1) or in Eq. (1.2), or whether more general nonlocal structures are phenomenologically acceptable. This would give a precious hint of the mechanism at work for the generation of the nonlocal terms. In this sense, the results of the present paper are meant to pave the way for future works aiming at understanding the origin of the nonlocal terms.

In this paper we study the generalization of nonlocal actions of the type (1.2), at the level quadratic in curvatures. As a basis for the curvature-square terms one could use $R_{\mu \nu \rho \sigma}^{2}, R_{\mu \nu}^{2}$ and $R^{2}$. However, for cosmological applications it is more convenient to trade the Riemann tensor $R_{\mu \nu \rho \sigma}$ for the Weyl tensor $C_{\rho \sigma \mu \nu}$, which in $d=3$ spatial dimensions is given by

$C_{\mu \nu \rho \sigma}=R_{\mu \nu \rho \sigma}-\left(g_{\mu[\rho} R_{\sigma] \nu}-g_{\nu[\rho} R_{\sigma] \mu}\right)+\frac{1}{3} g_{\mu[\rho} g_{\sigma] \nu} R$,

where $[\mu \nu]$ denotes the antisymmetrization over the indices, e.g. $T_{[\mu \nu]}=(1 / 2)\left(T_{\mu \nu}-T_{\nu \mu}\right)$. We will then consider an action of the form

$$
\begin{aligned}
S_{\mathrm{NL}}= & \frac{m_{\mathrm{Pl}}^{2}}{2} \int d^{4} x \sqrt{-g}\left[R-\mu_{1} R \frac{1}{\square^{2}} R-\mu_{2} C^{\mu \nu \rho \sigma} \frac{1}{\square^{2}} C_{\mu \nu \rho \sigma}\right. \\
& \left.-\mu_{3} R^{\mu \nu} \frac{1}{\square^{2}} R_{\mu \nu}\right],
\end{aligned}
$$

where $\mu_{1}, \mu_{2}$ and $\mu_{3}$ are independent parameters with dimension of squared mass. Another notable combination is the one that corresponds to the Gauss-Bonnet term,

$$
E=R_{\mu \nu \rho \sigma}^{2}-4 R_{\mu \nu}^{2}+R^{2}
$$

so instead of trading $R_{\mu \nu \rho \sigma} \square^{-2} R^{\mu \nu \rho \sigma}$ for $C^{\mu \nu \rho \sigma} \square^{-2} C_{\mu \nu \rho \sigma}$ one could trade it for the combination $R_{\mu \nu \rho \sigma} \square^{-2} R^{\mu \nu \rho \sigma}-$ $4 R_{\mu \nu} \square^{-2} R^{\mu \nu}+R \square^{-2} R$. However, the choice of basis in Eq. (1.4) is more convenient for cosmological applications, because the Weyl tensor vanishes in FRW.

It should be made clear that the space of all possible nonlocal theories is of course very large, and our choice by no means exhausts all possibilities. For instance, taking the transverse part of a tensor opens up new possibilities for writing nonlocal equations of motions, such as Eq. (1.1), which are not easily expressed in terms of an action. However, while the addition to the Einstein equations of a term proportional to $\left(g_{\mu \nu} \square^{-1} R\right)^{\mathrm{T}}$ gives a viable cosmological model, the addition of a term proportional to $\left(\square^{-1} R_{\mu \nu}\right)^{T}$ has been shown to be cosmologically nonviable, already at the background level $[12,14]$. Several other options are possible, which can be considered as nonlinear extensions of Eq. (1.2) or of Eq. (1.4). 
A particularly well-motivated extension of Eq. (1.2) is obtained by writing [29]

$$
S=\frac{m_{\mathrm{Pl}}^{2}}{2} \int d^{4} x \sqrt{-g}\left[R-\frac{1}{6} m^{2} R \frac{1}{\Delta_{4}} R\right],
$$

where

$$
\Delta_{4}=\square^{2}+2 R^{\mu \nu} \nabla_{\mu} \nabla_{\nu}-\frac{2}{3} R \square+\frac{1}{3}\left(\nabla^{\mu} R\right) \nabla_{\mu},
$$

is the so-called Paneitz operator. Indeed, this operator enters in the computation of the anomaly-induced effective action which, as discussed in Ref. [6], and as we will recall in the Conclusions, could be at the origin of these effective nonlocal IR modifications of GR. More generally, we could also replace $\square^{2}$ by $\Delta_{4}$ in Eq. (1.4).

In the present paper we study the cosmological consequences of the action (1.4), both at the level of background evolution and at the level of cosmological perturbations. The plan of the paper is as follows. In Sec. II we discuss the effect of the term $R_{\mu \nu} \square^{-2} R^{\mu \nu}$, and we will see that it is ruled out already at the background level. We then turn to the effect of adding the nonlocal Weyl term $C^{\mu \nu \rho \sigma} \square^{-2} C_{\mu \nu \rho \sigma}$ to the theory that already features the $R \square^{-2} R$ term. In Sec. III we show how to write the nonlocal Weyl term in local form introducing auxiliary fields. The cosmological consequences of the model are studied in Sec. IV. The nonlocal Weyl term does not affect the background evolution, but only the cosmological perturbations. The background evolution is therefore the same induced by the $R \square^{-2} R$ term, which is briefly reviewed in Sec. IV A. The decomposition into scalars, vectors and tensors of the perturbations associated to the nonlocal Weyl term is nontrivial, and we discuss it in Sec. IV B. Scalar perturbations are then discussed in Sec. IV C and tensor perturbations in Sec. IV D. We draw our conclusions in Sec. V. In the appendices we perform a more detailed comparison between analytic and numerical solutions of the equations in the scalar and tensor sector.

We use the Misner-Thorne-Wheeler conventions for the metric, Riemann tensor, etc., so in particular our signature is $(-,+,+,+)$, and we set $\hbar=c=1$. A prime will denote the derivative with respect to $x \equiv \log a$, where $a$ is the scale factor in FRW.

\section{THE $\boldsymbol{R}_{\mu \nu} \square^{-2} \boldsymbol{R}^{\mu \nu}$ TERM}

The term proportional to $\mu_{2}$ in the action (1.4) is quadratic in the Weyl tensor. Thus, in the equations of motion it gives a contribution linear in $C_{\mu \nu \rho \sigma}$, which vanishes in FRW. Therefore this term does not contribute to the background evolution, but only at the level of cosmological perturbation. To study first of all whether the model (1.4) is viable at the level of background evolution, we then only need to consider the action (1.4) with $\mu_{2}=0$. The corresponding equations of motion can be obtained in a standard way by localizing the action through the introduction of auxiliary scalar fields; see e.g. Refs. [3,11-13,30-34]. In particular, we introduce two auxiliary scalar fields

$$
U \equiv-\square^{-1} R, \quad S \equiv-\square^{-1} U=\square^{-2} R,
$$

and two auxiliary tensor fields,

$U_{\mu \nu} \equiv-\square^{-1} R_{\mu \nu}, \quad S_{\mu \nu} \equiv-\square^{-1} U_{\mu \nu}=\square^{-2} R_{\mu \nu}$.

These relations can be implemented at the level of the action by introducing four Lagrange multipliers $\zeta_{1}, \zeta_{2}, \zeta_{1}^{\mu \nu}$ and $\zeta_{2}^{\mu \nu}$, and writing

$$
\begin{aligned}
S_{\mathrm{NL}}= & \frac{m_{\mathrm{Pl}}^{2}}{2} \int d^{4} x \sqrt{-g}\left[R\left(1-\mu_{1} S\right)-\mu_{3} R_{\mu \nu} S^{\mu \nu}\right. \\
& -\zeta_{1}(\square U+R)-\zeta_{2}(\square S+U) \\
& \left.-\zeta_{1}^{\mu \nu}\left(\square U_{\mu \nu}+R_{\mu \nu}\right)-\zeta_{2}^{\mu \nu}\left(\square S_{\mu \nu}+U_{\mu \nu}\right)\right] .
\end{aligned}
$$

The variation with respect to the Lagrange multipliers and with respect to the auxiliary fields enforces the constraints $\zeta_{1}=\mu_{1} S, \quad \zeta_{2}=\mu_{1} U, \quad \zeta_{1}^{\mu \nu}=\mu_{3} S^{\mu \nu} \quad$ and $\quad \zeta_{2}^{\mu \nu}=\mu_{3} U^{\mu \nu}$. Varying the action (2.3) with respect to the metric, substituting these constraints and making use of Eqs. (2.1) and (2.2) afterwards, we find the following covariant equation:

$$
G_{\mu \nu}-\mu_{1} \mathcal{K}_{\mu \nu}+\mu_{3} \mathcal{I}_{\mu \nu}=8 \pi G T_{\mu \nu}
$$

where

$$
\begin{aligned}
\mathcal{K}_{\mu \nu}= & 2 S G_{\mu \nu}-2 \nabla_{\mu} \partial_{\nu} S-2 U g_{\mu \nu}+g_{\mu \nu} \partial_{\rho} S \partial^{\rho} U \\
& -(1 / 2) g_{\mu \nu} U^{2}-\left(\partial_{\mu} S \partial_{\nu} U+\partial_{\nu} S \partial_{\mu} U\right),
\end{aligned}
$$

is the part coming from the $R \square^{-2} R$ term, already found in Ref. [13], and

$$
\begin{aligned}
\mathcal{I}_{\mu \nu} & =\left\{-2 S_{\mu \alpha} G_{\nu}^{\alpha}-R S_{\mu \nu}-\frac{1}{2} \square S_{\mu \nu}-U_{\mu}^{\alpha} U_{\nu \alpha}+\frac{1}{2} g_{\mu \nu}\left(G^{\alpha \beta} S_{\alpha \beta}\right.\right. \\
& \left.+\frac{1}{2} U_{\alpha \beta} U^{\alpha \beta}+\frac{1}{2} R S_{\alpha}^{\alpha}-\nabla^{\rho} S^{\alpha \beta} \nabla_{\rho} U_{\alpha \beta}-\nabla_{\alpha} \nabla_{\beta} S^{\alpha \beta}\right) \\
& +\left[\frac{1}{2} \nabla_{\mu} U_{\alpha \beta} \nabla_{\nu} S^{\alpha \beta}+\nabla_{\beta} U_{\alpha}^{\beta} \nabla_{\mu} S_{\nu}^{\alpha}-S_{\nu}^{\alpha} \nabla_{\beta} \nabla_{\mu} U_{\alpha}^{\beta}-U_{\nu}^{\alpha} \square S_{\mu \alpha}\right. \\
& \left.\left.+U^{\alpha \beta} \nabla_{\beta} \nabla_{\mu} S_{\nu \alpha}-\nabla^{\beta} S_{\mu}^{\alpha} \nabla_{\nu} U_{\alpha \beta}+(U \leftrightarrow S)\right]\right\} \\
& +\{\mu \leftrightarrow \nu\} .
\end{aligned}
$$

We now specialize to a flat FRW metric, $d s^{2}=-d t^{2}+$ $a^{2}(t) d \mathbf{x}^{2}$ in $d=3$ spatial dimensions and in the presence of an energy-momentum tensor $T_{\mu \nu}=\left(\rho, a^{2}(t) p \delta_{i j}\right)$. Then, only the $(0,0)$ and the $(i, i)$ components of the field 
equations are nontrivial. It is convenient to introduce the variables $U=U_{0}^{0}+U_{i}^{i}, V=U_{0}^{0}-U_{i}^{i} / 3$ (where the sum over $i=1,2,3$ is understood). Then the nontrivial equations are

$$
\begin{aligned}
& \partial_{t}^{2} U+3 H \partial_{t} U=6 \partial_{t} H+12 H^{2}, \\
& \partial_{t}^{2} V+3 H \partial_{t} V-8 H^{2} V=2 \partial_{t} H,
\end{aligned}
$$

where $\partial_{t}$ is the derivative with respect to cosmic time. It is easy to see that the equation for $V$ contains growing modes. To this purpose it is convenient to parametrize the time evolution in terms of the variable $x \equiv \log a$. Then Eq. (2.8) reads

$$
V^{\prime \prime}+V^{\prime}(3+\zeta)-8 V=-2 \zeta
$$

where $f^{\prime} \equiv d f / d x$ and $\zeta(x)=h^{\prime}(x) / h(x)$. During radiation domination (RD), matter domination (MD) and a de Sitter phase $\zeta(x)$ can be approximated by a constant $\zeta_{0}$, with $\zeta_{0}=\{-2,-3 / 2,0\}$, respectively. The homogeneous equation associated to Eq. (2.9) is the same encountered in the nonlocal model introduced in Ref. [11] and further analyzed in Ref. [14]. The solution in the approximation $\zeta(x)=\zeta_{0}$ is

$$
V(x)=\frac{\zeta_{0}}{4}+v_{0} e^{\beta_{+} x}+v_{1} e^{\beta_{-} x},
$$

with $2 \beta_{ \pm}=-\left(3+\zeta_{0}\right) \pm \sqrt{\left(3+\zeta_{0}\right)^{2}+32}$. We see that $\beta_{+}>0$ both in MD and RD, which leads to a growing solution for $V$. This affects the whole cosmological evolution, since the function $V$ is coupled to all other variables through its contribution to $\mathcal{I}_{00}$. So, similarly to the model discussed in Ref. [11] and in Appendix A of Ref. [14], based on a term $\left(\square^{-1} G_{\mu \nu}\right)^{T}$ in the equations of motion, there is no stable cosmological evolution. In particular, if we start close to a standard FRW solution at early times, the evolution quickly departs from it and explodes. Thus, the term $\mu_{3} R^{\mu \nu} \square^{-2} R_{\mu \nu}$ is not cosmologically viable (unless of course the parameter $\mu_{3}$ is tuned to be extremely suppressed with respect to $\mu_{1}$; imposing such a condition on a phenomenological model is quite unnatural so we do not further explore this possibility).

This result is similar to the one found in Ref. [35], where it was shown that a term $R^{\mu \nu} \square^{-1} R_{\mu \nu}$ also produces instabilities in the cosmological evolution. Observe that the latter term is rather of the Deser-Woodard type, i.e. of the form $R^{\mu \nu} f\left(\square^{-1} R_{\mu \nu}\right)$, with a dimensionless function $f$ and no explicit mass scale $m$. However, in both cases the instability is ultimately due to the form of the $\square^{-1}$ operator on the tensor $R_{\mu \nu}$.

\section{NONLOCAL WEYL-SQUARE TERM AND AUXILIARY FIELDS}

The study of the background evolution shows that the term $R^{\mu \nu} \square^{-2} R_{\mu \nu}$ cannot be present in a viable model, i.e. $\mu_{3}=0$, but tells us nothing about the nonlocal Weyl-square term, since the latter does not contribute to the background evolution. To see whether this term is viable we must move one step forward, and see if the cosmological perturbations in the presence of this term are well behaved. We then consider the model with the action

$S_{\mathrm{NL}}=\frac{m_{\mathrm{Pl}}^{2}}{2} \int d^{4} x \sqrt{-g}\left[R-\mu_{1} R \frac{1}{\square^{2}} R-\mu_{2} C^{\mu \nu \alpha \beta} \frac{1}{\square^{2}} C_{\mu \nu \alpha \beta}\right]$.

The equations of motion of this model can again be derived by introducing auxiliary scalar fields. In this case we need again two auxiliary scalar fields

$$
U=-\square^{-1} R, \quad S=-\square^{-1} U=\square^{-2} R,
$$

as well as two auxiliary fields with the symmetry properties of the Weyl tensor, defined as

$$
\begin{aligned}
& U_{\mu \nu \alpha \beta}=-\square^{-1} C_{\mu \nu \alpha \beta}, \\
& S_{\mu \nu \alpha \beta}=-\square^{-1} U_{\mu \nu \alpha \beta}=\square^{-2} C_{\mu \nu \alpha \beta} .
\end{aligned}
$$

Proceeding as in Sec. II, we find the following covariant equation:

$$
\begin{aligned}
G_{\mu \nu} & -\mu_{1} \mathcal{K}_{\mu \nu}-2 \mu_{2} S_{\mu \alpha \nu \beta} R^{\beta \alpha}-2 \mu_{2}\left(\nabla^{\alpha} \nabla^{\beta}+\nabla^{\beta} \nabla^{\alpha}\right) S_{\mu \alpha \nu \beta} \\
- & \mu_{2} \mathcal{P}_{\mu \nu}=8 \pi G T_{\mu \nu},
\end{aligned}
$$

where $\mathcal{K}_{\mu \nu}$ is the same as in Eq. (2.5), and

$$
\begin{aligned}
\mathcal{P}_{\mu \nu}= & \left\{S_{\mu \sigma \alpha \beta} C_{\nu}^{\sigma \alpha \beta}-\frac{1}{4} g_{\mu \nu} C^{\alpha \beta \rho \sigma} S_{\alpha \beta \rho \sigma}+\frac{1}{4} g_{\mu \nu} \square\left(S_{\alpha \beta \rho \sigma} U^{\alpha \beta \rho \sigma}\right)\right. \\
& -\left(\boldsymbol{\nabla}_{\mu} S_{\alpha \beta \rho \sigma}\right)\left(\nabla_{\nu} U^{\alpha \beta \rho \sigma}\right)-2\left[\left(\nabla^{\sigma} \nabla_{\mu} U_{\nu}^{\alpha \rho \sigma}\right) S_{\sigma \alpha \rho \sigma}\right. \\
& \left.-\left(\nabla_{\beta} \nabla_{\mu} U^{\beta \sigma \rho \alpha}\right) S_{\nu \sigma \rho \alpha}+(U \leftrightarrow S)\right] \\
& -2\left[\left(\nabla_{\sigma} S^{\sigma \rho \alpha \beta}\right)\left(\nabla_{\mu} U_{\nu \rho \alpha \beta}\right)-\left(\nabla_{\beta} S_{\mu \alpha \rho \sigma}\right)\left(\nabla_{\nu} U^{\beta \alpha \rho \sigma}\right)\right. \\
& +(U \leftrightarrow S)]\}+\{\mu \leftrightarrow \nu\} .
\end{aligned}
$$

The set of equations describing the model is therefore given by Eqs. (3.4) and (3.5), together with

$$
\square U=-R, \quad \square S=-U,
$$

and

$$
\square U_{\mu \nu \alpha \beta}=-C_{\mu \nu \alpha \beta}, \quad \square S_{\mu \nu \alpha \beta}=-U_{\mu \nu \alpha \beta} .
$$


As discussed in detail in Refs. [3,21,32-34], transforming a nonlocal equation such as $U=-\square^{-1} R$ into the local equation $\square U=-R$ introduces spurious solutions, given by the most general solution of the associated homogeneous equation $\square U=0$. These spurious solutions are eliminated by fixing once and for all the boundary condition of the equation $\square U=0$ (which corresponds to choosing once and for all the definition of the $\square^{-1}$ operator). As in Refs. [12-14,18], we will fix the boundary conditions by requiring that the auxiliary fields vanish deep in $\mathrm{RD}^{2}$

We also observe that, since the modified Einstein equations (3.4) are obtained by varying an action invariant under diffeomorphisms, the energy-momentum tensor $T_{\mu \nu}$ is automatically covariantly conserved, $\nabla_{\mu} T_{\nu}^{\mu}=0$, as can also be explicitly checked by taking the covariant derivative of the right-hand side of Eq. (3.4) and using the equations of motion for the auxiliary fields.

\section{COSMOLOGY WITH THE NONLOCAL WEYL-SQUARE TERM}

\section{A. Background evolution}

At the level of the background, the evolution is the same as in the model $\mu_{2}=0$, already studied in Ref. [13]. For later use, let us recall the main results. We consider a flat FRW metric, and we use conformal time, $d s^{2}=$ $a^{2}(\eta)\left(-d \eta^{2}+d \mathbf{x}^{2}\right)$. Then, the Einstein equations and the evolution of the background configurations of the auxiliary fields $U$ and $S$, Eq. (3.6), can be recast in the form

$$
\begin{gathered}
h^{2}=\frac{\Omega_{M} e^{-3 x}+\Omega_{R} e^{-4 x}+\left(\gamma_{1} / 4\right) \bar{U}^{2}}{1+\gamma_{1}\left(-3 \bar{V}^{\prime}-3 \bar{V}+\frac{1}{2} \bar{V}^{\prime} \bar{U}^{\prime}\right)}, \\
\bar{U}^{\prime \prime}+(3+\zeta) \bar{U}^{\prime}=6(2+\zeta), \\
\bar{V}^{\prime \prime}+(3+\zeta) \bar{V}^{\prime}=\bar{U} h^{-2},
\end{gathered}
$$

where the bar over $U$ and $V$ denotes their background value, the prime is the derivative with respect to $x=\log a$, and $h=\mathcal{H} / \mathcal{H}_{0}=H / H_{0}$, where $H=(1 / a) d a / d t$. As usual, $\Omega_{R}$ and $\Omega_{M}$ are the energy fractions of radiation and matter at present time normalized with respect to $\rho_{0}=3 H_{0}^{2} /(8 \pi G)$. We also introduced the notation $\bar{V} \equiv H_{0}^{2} \bar{S}$, as well as $\zeta \equiv h^{\prime} / h$ and

\footnotetext{
${ }^{2}$ In general, if the homogeneous solutions for the auxiliary fields during RD only have decaying modes, as is the case for the models (1.1) and (1.2), choosing the initial conditions that correspond to setting them to zero, e.g. $U_{\text {hom }}=0$, is very natural, because this solution is an attractor. If in contrast there are growing modes, one must first of all check whether these instabilities are fatal for the viability of the model, and in any case the results will depend on the initial conditions chosen.
}

$$
\gamma_{1} \equiv \frac{2 \mu_{1}}{3 H_{0}^{2}}
$$

Equations (4.1)-(4.3) are a closed system and can be solved numerically given the initial conditions on the auxiliary fields $\bar{U}, \bar{V}$ fields and their first derivative. In particular, we assume $\bar{U}=\bar{V}=\bar{U}^{\prime}=\bar{V}^{\prime}=0$, at an initial time deep in $\mathrm{RD}$. It is also useful to define an effective dark energy (DE) density $\rho_{\mathrm{DE}}$, rewriting the modified Friedmann equation (4.1) as

$$
h^{2}(x)=\Omega_{M} e^{-3 x}+\Omega_{R} e^{-4 x}+\frac{\rho_{\mathrm{DE}}}{\rho_{0}} .
$$

Similarly, we can define an effective DE pressure $p_{\mathrm{DE}}$ from the trace of the $(i j)$ modified Einstein equations, and the corresponding equation-of-state parameter $w_{\mathrm{DE}} \equiv p_{\mathrm{DE}} / \rho_{\mathrm{DE}}$.

In $\Lambda$ CDM one typically uses, as independent cosmological parameters, the baryon density today $\omega_{b}=\Omega_{b} h_{0}^{2}$, the cold dark matter density $\omega_{c}=\Omega_{\mathrm{c}} h_{0}^{2}$, the Hubble parameter today $H_{0}=h_{0} \times 100 \mathrm{~km} \mathrm{~s}^{-1} \mathrm{Mpc}^{-1}$, the amplitude of scalar perturbations $A_{s}$, the scalar spectrum index $n_{s}$ and the redshift at which the Universe is half-reionized $z_{\text {re }}$. The dark energy density $\Omega_{\Lambda}$ is then a derived parameter, fixed by the flatness condition. In the nonlocal model with $\mu_{2}=0$ the situation is similar. We can use the same set of independent cosmological parameters and then $\mu_{1}$, or equivalently $\gamma_{1}$, is a derived parameter, fixed again from the condition $\Omega_{\text {tot }}=1$. The corresponding comparison with data for the $R \square^{-2} R$ model has been performed in Ref. [18], implementing the cosmological perturbations of the nonlocal models into a Boltzmann code and performing parameter estimation. The best-fit values for $\omega_{c}, \omega_{b}$ and $h_{0}$, using Planck $2013 \mathrm{CMB}$ data, JLA SNe and BAO are $\omega_{c}=0.1204(14), \omega_{b}=2.197(25) \times 10^{-2}$ and $h_{0}=0.709(7)$, corresponding to $\Omega_{M}=\left(\omega_{c}+\omega_{b}\right) / h_{0}^{2}=0.283(9)$. The value of $\gamma_{1}$ is then fixed so to reproduce this best-fit value, which gives ${ }^{3} \gamma_{1} \simeq 9.286 \times 10^{-3}$.

\section{B. Scalar-vector-tensor decomposition of the perturbations}

We now turn to the study of the cosmological perturbations of this nonlocal model. We consider the following metric, in conformal time and longitudinal gauge:

\footnotetext{
${ }^{3}$ In principle the value of $\gamma_{1}$ is determined by trial and error so that the evolution produces the required value of $\Omega_{\mathrm{DE}}$ and therefore of $\Omega_{M}$ at the present time $x=0$. However, in the region $\Omega_{M} \in[0.20,0.35]$, the required value of $\gamma_{1}$ is accurately reproduced by the fit $\gamma_{1}=0.0103959+0.00687851 \Omega_{M}-$ $0.0598026 \Omega_{M}^{2}+0.094128 \Omega_{M}^{3}-0.0624636 \Omega_{M}^{4} \quad$ [16]. Observe also that this value of $\gamma$ has been obtained using the Planck 2013 data. An updated analysis using Planck 2015 data will be presented in Ref. [19].
} 


$$
\begin{aligned}
d s^{2}= & a^{2}(\eta)\left\{-(1+2 \Psi) d \eta^{2}+2 w_{i} d \eta d x^{i}\right. \\
& \left.+\left[(1+2 \Phi) \delta_{i j}+2 h_{i j}\right] d x^{i} d x^{j}\right\},
\end{aligned}
$$

where $\Phi$ and $\Psi$ describe the scalar perturbations, the transverse vector $w_{i}$ describes vector perturbations, and the transverse-traceless tensor $h_{i j}$ describes tensor perturbations. Let us recall that, for a generic anisotropic fluid, at first order in perturbation theory we have $T_{0}^{0}=-(\bar{\rho}+\delta \rho)$, $T_{i}^{0}=(\bar{\rho}+\bar{p}) v_{i}$ and $T_{j}^{i}=(\bar{p}+\delta p) \delta_{j}^{i}+\Sigma_{j}^{i}$, where the density and the pressure have been perturbed around their background values, $\rho=\bar{\rho}+\delta \rho$ and $p \equiv \bar{p}+\delta p$. The pressure perturbation is proportional to the density perturbation, $\delta p=c_{s}^{2} \delta \rho$, where $c_{s}^{2}$ is the speed of sound of the fluid. We define as usual $\delta \equiv \delta \rho / \bar{\rho}$ and $\theta \equiv \delta^{i j} \partial_{i} v_{j}$. The anisotropic stress $\Sigma_{j}^{i}$ is a symmetric and traceless tensor, $\Sigma_{i}^{i}=0$. We consider the Universe filled with radiation and nonrelativistic matter, and therefore in the following we will set $\Sigma_{j}^{i}=0$. We expand the auxiliary fields $U$ and $S$ around some background configuration as $U=\bar{U}+\delta U$ and $S=\bar{S}+\delta S$. In principle one can do the same with $U_{\mu \nu \alpha \beta}$ and $S_{\mu \nu \alpha \beta}$, parametrizing perturbations around some background configuration $\bar{U}_{\mu \nu \alpha \beta}$ and $\bar{S}_{\mu \nu \alpha \beta}$. However, as we will explicitly show later, from the definitions (3.3) it turns out that the background configurations $\bar{U}_{\mu \nu \alpha \beta}$ and $\bar{S}_{\mu \nu \alpha \beta}$ are vanishing on a cosmological FRW background. Therefore, the auxiliary tensor fields $U_{\mu \nu \alpha \beta}$ and $S_{\mu \nu \alpha \beta}$ do not enter the background equations and only affect the perturbations. This is consistent with what was already observed: at the background level the model (3.1) is indistinguishable from the one with $\mu_{2}=0$. We also observe that, as long as we are interested in perturbations of first order in the equations of motion, the term $\mathcal{P}_{\mu \nu}$ in Eq. (3.4) does not contribute, since it is quadratic in the tensor fields $U_{\mu \nu \alpha \beta}$ and $S_{\mu \nu \alpha \beta}$, which have vanishing background configurations on a FRW background for the metric. Computing the Weyl tensor in terms of the metric perturbations we find, to first order

$$
\begin{aligned}
C_{i 0 j}^{0}= & -\frac{1}{2} D_{i j}(\Psi-\Phi)-\frac{1}{2} \partial_{\eta} w_{i j}+\frac{1}{2}\left(\partial_{\eta}^{2}+\nabla^{2}\right) h_{i j}, \\
C^{0}{ }_{i j k}= & \left(\nabla_{k} w_{i j}-\nabla_{j} w_{i k}\right)+\frac{1}{4} \nabla^{2}\left(\delta_{i j} w_{k}-\delta_{i k} w_{j}\right) \\
& +\left(\nabla_{j} \partial_{\eta} h_{i k}-\nabla_{k} \partial_{\eta} h_{i j}\right),
\end{aligned}
$$

where

$$
D_{i j} \equiv \boldsymbol{\nabla}_{i} \boldsymbol{\nabla}_{j}-\frac{1}{3} \delta_{i j} \boldsymbol{\nabla}^{2}, \quad w_{i j} \equiv \boldsymbol{\nabla}_{(i} w_{j)},
$$

and, for any tensor $A_{\mu \nu}$, we define $A_{(\mu \nu)} \equiv(1 / 2)\left(A_{\mu \nu}+A_{\nu \mu}\right)$ and $A_{[\mu \nu]} \equiv(1 / 2)\left(A_{\mu \nu}-A_{\nu \mu}\right)$. The other components of the Weyl tensor follow from the symmetry relations $C_{\mu \nu \alpha \beta}=$ $C_{[\mu \nu][\alpha \beta]}=C_{\alpha \beta \mu \nu}$ and $C_{\mu\{\nu \alpha \beta\}}=0$ (where, for any tensor
$\left.A_{\mu \nu \rho}, A_{\{\mu \nu \rho\}} \equiv A_{\mu \nu \rho}+A_{\nu \rho \mu}+A_{\rho \mu \nu}\right)$, which in $3+1$ dimensions also imply

$C_{i j k l}=\delta_{j l} C_{0 i 0 k}+\delta_{i k} C_{0 j 0 l}-\delta_{i l} C_{0 j 0 k}-\delta_{j k} C_{0 i 0 l}$.

Note that, since we are retaining only terms of first order in the metric perturbations, the components of the Weyl tensor may be raised and lowered using the unperturbed metric. For the independent components of the $U_{\mu \nu \alpha \beta}$ tensor, i.e. $U_{0 i 0 j}$ and $U_{0 i j k}$, Eq. (3.6) on a FRW background is equivalent to the following set of coupled differential equations:

$$
\begin{aligned}
& \partial_{\eta}^{2} U_{0 i j k}-6 \mathcal{H} \partial_{\eta} U_{0 i j k}+\left(2 \mathcal{H}^{2}-4 \partial_{\eta} \mathcal{H}\right) U_{0 i j k} \\
& =\nabla^{2} U_{0 i j k}-a^{2} C_{0 i j k}+2 \mathcal{H} \delta_{i j} \partial_{q} U_{0 q 0 k}-2 \mathcal{H} \delta_{i k} \partial_{q} U_{0 q 0 j} \\
& \quad-4 \mathcal{H} \partial_{j} U_{0 i 0 k}+4 \mathcal{H} \partial_{k} U_{0 i 0 j}, \\
& \partial_{\eta}^{2} U_{0 i 0 j}-6 \mathcal{H} \partial_{\eta} U_{0 i 0 j}+\left(2 \mathcal{H}^{2}-4 \partial_{\eta} \mathcal{H}\right) U_{0 i 0 j} \\
& \quad=\nabla^{2} U_{0 i 0 j}+2 \mathcal{H} \partial_{q} U_{0 j i q}+2 \mathcal{H} \partial_{q} U_{0 i j q}-a^{2} C_{0 i 0 j},
\end{aligned}
$$

where, in the first equation, we used the fact that the tensor $U_{i j k l}$ satisfies the same symmetry relation as the Weyl tensor, so in particular also the analog of the relation (4.10). Analogously one has, for $S_{\mu \nu \rho \sigma}$,

$$
\begin{aligned}
& \partial_{\eta}^{2} S_{0 i j k}-6 \mathcal{H} \partial_{\eta} S_{0 i j k}+\left(2 \mathcal{H}{ }^{2}-4 \partial_{\eta} \mathcal{H}\right) S_{0 i j k} \\
&= \nabla^{2} S_{0 i j k}-a^{2} U_{0 i j k}+2 \mathcal{H} \delta_{i j} \partial_{q} S_{0 q 0 k}-2 \mathcal{H} \delta_{i k} \partial_{q} S_{0 q 0 j} \\
&-4 \mathcal{H} \partial_{j} S_{0 i 0 k}+4 \mathcal{H} \partial_{k} S_{0 i 0 j},
\end{aligned}
$$

$$
\begin{aligned}
& \partial_{\eta}^{2} S_{0 i 0 j}-6 \mathcal{H} \partial_{\eta} S_{0 i 0 j}+\left(2 \mathcal{H}^{2}-4 \partial_{\eta} \mathcal{H}\right) S_{0 i 0 j} \\
& \quad=\nabla^{2} S_{0 i 0 j}+2 \mathcal{H} \partial_{q} S_{0 j i q}+2 \mathcal{H} \partial_{q} S_{0 i j q}-a^{2} U_{0 i 0 j} .
\end{aligned}
$$

We observe that, as already mentioned in Sec. IV, if we solve Eqs. (4.12)-(4.13) for the background configurations of the auxiliary fields, the source term $C_{0 i j k}$ vanishes and we get as a solution $\bar{U}_{\mu \nu \alpha \beta}=0$ and $\bar{S}_{\mu \nu \alpha \beta}=0$.

Note that all equations above involve the same differential operator in conformal time

$$
\mathcal{T}[X] \equiv \partial_{\eta}^{2} X-6 \mathcal{H} \partial_{\eta} X+\left(2 \mathcal{H}^{2}-4 \partial_{\eta} \mathcal{H}\right) X,
$$

which may allow for unstable solutions. This can be seen explicitly by using $x=\log a$ as a time variable. Then the differential operator becomes

$\mathcal{T}[X] \rightarrow \hat{\mathcal{T}}[X] \equiv \mathcal{H}^{2}\left[X^{\prime \prime}+(\zeta-5) X^{\prime}-2(2 \zeta+1) X\right]$.

As in Sec. II, we can take $\zeta(x) \simeq \zeta_{0}$ as approximatively constant in the various cosmological epochs, with 
$\zeta_{0}=\{-2,-3 / 2,0\}$ in radiation, matter and de Sitter, respectively. One then finds that in RD there is a mode growing as $e^{6 x}=a^{6}$ which might suggest that the model is badly unstable; however, we will see that the auxiliary fields suffering this instability enter in the Einstein equations with a suppression factor which is just $a^{-6}$, so an explicit inspection of the Einstein equations is necessary in order to understand the behavior of the system.

We now parametrize the various components of $U_{\mu \nu \alpha \beta}$ and $S_{\mu \nu \alpha \beta}$ in terms of scalar, vector and tensor degrees of freedom. The five degrees of freedom characterizing the symmetric and traceless tensors $U_{0 i 0 j}$ and $S_{0 i 0 j}$ can be decomposed in the usual way into a scalar, a transverse vector, and a transverse-traceless symmetric tensor:

$$
\begin{gathered}
U_{0 i 0 j}=D_{i j} u+\nabla_{(i} u_{j)}+u_{i j}, \\
S_{0 i 0 j}=D_{i j} s+\nabla_{(i} s_{j)}+s_{i j},
\end{gathered}
$$

with $\boldsymbol{\nabla}_{i} u^{i}=\nabla_{i} s^{i}=u_{i}^{i}=s_{i}^{i}=\nabla_{i} u^{i j}=\nabla_{i} s^{i j}=0$. For the other components, one may notice that $S_{0 i j k}$ enters in the Einstein equations only through the combinations $S_{0(i j) k, k} \equiv S_{i j}$, and therefore $U_{0 i j k}$ only enters through the combination $U_{0(i j) k, k} \equiv U_{i j}$. These combinations are symmetric, traceless and with one vanishing longitudinal component $\left(S_{i j, i j}=0\right)$, so they only carry four degrees of freedom (the scalar is missing). Then, they are naturally parametrized as

$$
S_{i j}=\nabla_{(i} \sigma_{j)}+\sigma_{i j}
$$

Observe that $\boldsymbol{\nabla}_{i} \boldsymbol{\nabla}_{j} S_{i j}=0$ to first order in perturbation theory since, on FRW, the Christoffel symbol $\Gamma_{i j}^{k}=0$. Similarly, we write

$$
U_{i j}=\nabla_{(i} v_{j)}+v_{i j},
$$

with $\boldsymbol{\nabla}_{i} v^{i}=\nabla_{i} \sigma^{i}=v_{i}^{i}=\sigma_{i}^{i}=\nabla_{i} v^{i j}=\nabla_{i} \sigma^{i j}=0$. We now have all the elements for writing down the Einstein equations to first order in the cosmological perturbations, and decomposing them in the usual way into scalar, vector and tensor components. We also go to Fourier space, denoting the comoving momentum by $k$. We introduce $\hat{k} \equiv k /(a H)$, and we use $x=\log a$ as a time variable, with $d f / d x=f^{\prime}$. It is also useful to introduce the dimensionless couplings

$$
\gamma_{1} \equiv \frac{2 \mu_{1}}{3 H_{0}^{2}}, \quad \gamma_{2} \equiv \frac{2 \mu_{2}}{3 H_{0}^{2}}
$$

and to rescale the fields as follows:

$$
\begin{gathered}
V=H_{0}^{2} S, \quad \hat{s}=H_{0}^{4} s, \quad \hat{u}=H_{0}^{2} u, \\
\hat{s}_{i j} \equiv H_{0}^{2} s_{i j}, \quad \hat{\sigma}_{i j} \equiv H_{0} \sigma_{i j}, \quad \hat{v}_{i j} \equiv \frac{v_{i j}}{H_{0}} .
\end{gathered}
$$

\section{Scalar sector}

In the scalar sector, we can take as independent equations the trace and the traceless parts of the $(i j)$ component of the perturbed Einstein equations. The former gives

$$
\begin{aligned}
& \left(1-3 \gamma_{1} \bar{V}\right)\left[\Phi^{\prime \prime}+(\zeta+3) \Phi^{\prime}-\Psi(3+2 \zeta)-\Psi^{\prime}+\frac{\hat{k}^{2}}{3}(\Psi+\Phi)\right] \\
& -\frac{3}{2} \gamma_{1}\left[\frac{1}{2 h^{2}} \bar{U} \delta U-2 \Psi \bar{V}^{\prime \prime}+\left[2 \Phi^{\prime}-2(\zeta+2) \Psi-\Psi^{\prime}-\Psi \bar{U}^{\prime}\right] \bar{V}^{\prime}\right. \\
& +\delta V^{\prime \prime}+(\zeta+2) \delta V^{\prime}+\frac{2 \hat{k}^{2}}{3} \delta V+(3+2 \zeta) \delta V \\
& \left.+\frac{1}{2}\left(\bar{U}^{\prime} \delta V^{\prime}+\bar{V}^{\prime} \delta U^{\prime}\right)\right]+\frac{2}{3} \frac{\gamma_{2}}{a^{2}} h^{2} \hat{k}^{4} s=-4 \pi G \frac{\delta p}{H^{2}},
\end{aligned}
$$

and the latter

$$
\begin{aligned}
\left(1-3 \gamma_{1} \bar{V}\right) \Psi= & -\left(1-3 \gamma_{1} \bar{V}\right) \Phi+3 \gamma_{1} \delta V \\
& -\frac{6 \gamma_{2}}{a^{2}} h^{2}\left[\hat{s}^{\prime \prime}+(\zeta-3) \hat{s}^{\prime}-\left(\frac{5}{4} \zeta-2\right) \hat{s}+\frac{\hat{k}^{2}}{3} \hat{s}\right] .
\end{aligned}
$$

As usual, the (00) component of the Einstein equation is not independent from these equations. The equations for the auxiliary fields can be written as

$$
\begin{aligned}
\delta U^{\prime \prime} & +(3+\zeta) \delta U^{\prime}+\hat{k}^{2} \delta U-2 \Psi \bar{U}^{\prime \prime}-\left[2(3+\zeta) \Psi+\Psi^{\prime}-3 \Phi^{\prime}\right] \bar{U}^{\prime} \\
& =2 \hat{k}^{2}(\Psi+2 \Phi)+6\left[\Phi^{\prime \prime}+(\zeta+4) \Phi^{\prime}\right]-6\left[\Psi^{\prime}+2(\zeta+2) \Psi\right],
\end{aligned}
$$

$$
\begin{gathered}
\delta V^{\prime \prime}+(\zeta+3) \delta V^{\prime}+\hat{k}^{2} \delta V-2 \Psi \bar{V}^{\prime \prime} \\
-\left[2(\zeta+3) \Psi+\Psi^{\prime}-3 \Phi^{\prime}\right] \bar{V}^{\prime}=\frac{\delta U}{h^{2}}, \\
\hat{s}^{\prime \prime}+(\zeta-5) \hat{s}^{\prime}-2(2 \zeta+1) \hat{s}+\hat{k}^{2} s=-\frac{1}{h^{2}} \hat{u}, \\
\hat{u}^{\prime \prime}+(\zeta-5) \hat{u}^{\prime}-2(2 \zeta+1) \hat{u}+\hat{k}^{2} u=-\frac{a^{2}}{2} \frac{1}{h^{2}}(\Psi-\Phi) .
\end{gathered}
$$

From the covariant conservation of the energy-momentum tensor, $\nabla^{\mu} T_{\mu \nu}=0$, we find

$$
\delta^{\prime}=-\left(3 \Phi^{\prime}+\hat{\theta}\right)(1+w)-3 \delta\left(c_{s}^{2}-w\right),
$$


$\hat{\theta}^{\prime}=-\left(2-3 w+\zeta+\frac{w^{\prime}}{1+w}\right) \hat{\theta}+\hat{k}^{2}\left(\Psi+\sigma+\frac{c_{s}^{2}}{1+w} \delta\right)$,

where $w$ is defined by $\bar{p}=w \bar{\rho}$, while $c_{s}^{2}$ from $\delta p=c_{s}^{2} \delta \rho{ }^{4}$ These equations are independent of the specific dark energy content, since they simply express the conservation of $T_{\mu \nu}$. For matter-radiation fluids with no energy exchange among them, we have as usual

$$
\begin{aligned}
& \delta_{M}^{\prime}=-\left(3 \Phi^{\prime}+\hat{\theta}_{M}\right), \\
& \hat{\theta}_{M}^{\prime}=-(2+\zeta) \hat{\theta}_{M}+\hat{k}^{2} \Psi \\
& \delta_{R}^{\prime}=-\frac{4}{3}\left(3 \Phi^{\prime}+\hat{\theta}_{R}\right) \\
& \hat{\theta}_{R}^{\prime}=-(1+\zeta) \hat{\theta}_{R}+\hat{k}^{2}\left(\Psi+\frac{\delta_{R}}{4}\right) .
\end{aligned}
$$

We set to zero the amplitude of the auxiliary fields and of their derivatives at an initial time deep into RD. For the matter and metric variables, we take the standard adiabatic initial conditions expanded to second order [see e.g. Ref. [36], or Eqs. (6.1)-(6.3) of Ref. [16]]. For the spectral index $n_{s}$ and for the amplitude of the gravitational potential $\delta_{H}$, we take the values, $n_{s} \simeq 0.96, \delta_{H}^{2} \simeq 3.2 \times 10^{-10}$. We can now solve the set of coupled differential equations (4.23)-(4.28), (4.31)-(4.34), for different values of $k$.

Let us recall that the modes relevant for the comparison with $\mathrm{CMB}$ and with structure formation data correspond roughly to the range $k=\left(10^{-3}-10\right) h / \mathrm{Mpc}$, and that in $\Lambda \mathrm{CDM}$ modes with $k \lesssim 0.3 \mathrm{~h} / \mathrm{Mpc}$ remain in the linear regime up to the present epoch, while modes with $k \gtrsim$ $0.3 \mathrm{~h} / \mathrm{Mpc}$ become nonlinear. In terms of the quantity $k / H_{0}$, the range relevant for $\mathrm{CMB}$ and structure formation is roughly $k / H_{0} \in\left[3,3 \times 10^{4}\right]$, and nonlinear scales correspond to $k / H_{0} \gtrsim 900$.

Our numerical results are shown in Fig. 1. In this figure we show the results for three different values of the (reduced) comoving wavelength $\chi=1 / k=$ $\{20,93,1000\} \mathrm{Mpc}$. These values are representative of different kinds of behaviors. Indeed, the mode with $\chi=$ $20 \mathrm{Mpc}$ (i.e. $k / H_{0} \simeq 214$, or $k \simeq 0.071 \mathrm{Mpc} / h_{0}$ ) reenters the horizon deep in RD. The mode with $\chi=93 \mathrm{Mpc}$ (i.e. $k / H_{0} \simeq 46$, or $k \simeq 0.015 \mathrm{Mpc} / h_{0}$ ) is the one that (when $h_{0}=0.7$ and $\Omega_{M}=0.3$ ) reenters the horizon exactly at the RD-MD transition. Finally, the mode with $\chi=1$ Gpc (i.e. $k / H_{0} \simeq 4.3$, or $k \simeq 0.001 \mathrm{Mpc} / h_{0}$ ) reenters the horizon only near the present epoch.

\footnotetext{
${ }^{4}$ Recall that, for a barotropic fluid, $c_{s}^{2}=w+\left(\bar{\rho} / \bar{\rho}^{\prime}\right) w^{\prime}$. The term $w^{\prime}$ is only important at the transition between different phases. In a given phase, such as RD or MD, when $w$ is constant to good accuracy, $c_{s}^{2} \simeq w$.
}

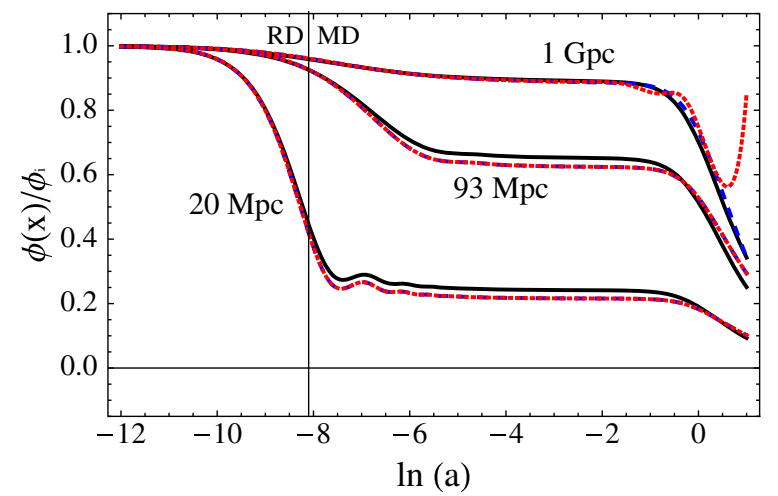

FIG. 1. Scalar perturbations for three different comoving reduced wavelengths $\chi=1 / k$. For each wavelength we plot the result for $\Lambda$ CDM (black solid line), the pure $R \square^{-2} R$ model with $\mu_{1}=0.014 H_{0}^{2}$ and $\mu_{2}=0$ (blue, dashed), and the model with $\mu_{1}=\mu_{2}=0.014 H_{0}^{2}$ (red, dotted). For $\chi=20 \mathrm{Mpc}$ and for $\chi=93 \mathrm{Mpc}$ the blue and the red curves are basically indistinguishable on this scale, while for $\chi=1 \mathrm{Gpc}$ the blue and black lines are almost indistinguishable.

For each of these wavelengths we show the standard $\Lambda \mathrm{CDM}$ result (black solid line), the result for the pure $R \square^{-2} R$ model (blue, dashed line) with $\mu_{1}=0.014 H_{0}^{2}$, which corresponds to the best-fit value for $\gamma$ given in Sec. IV A, and the result for the model with the Weyl-square term, where we set for definiteness $\mu_{1}=\mu_{2}=0.014 H_{0}^{2}$ (red, dotted). We see that, compared to the pure $R \square^{-2} R$ model, and up to the present time, only the largest modes are affected by the addition of the Weyl-square term. Even for these very long wavelengths, which are the largest observable with CMB anisotropies, the difference with the $R \square^{-2} R$ model is negligible during $\mathrm{MD}$, and only shows up as a difference of at most (1-2)\% percent near the recent epoch, $x=0$ (although scalar perturbations in the Weyl model will start to show an instability in the cosmological future, $x>0$ ).

This means that, compared to the pure $R \square^{-2} R$ model, the addition of the Weyl-square term has basically no effect on $\mathrm{SNe}, \mathrm{BAO}$, and structure formation data while, in the $\mathrm{CMB}$, it only affects the late integrated Sachs-Wolfe (ISW) effect, just at the level of order (1-2)\% percent. The late ISW effect only contributes to the very low multipoles, and even for these multipoles it is subleading with respect to the (nonintegrated) Sachs-Wolfe effect. Thus, among all cosmological observations testing scalar perturbations, the inclusion of the Weyl-square term will only affect the lowest CMB multipoles, and even for these it will only result in an overall deviation of the $C_{l}$ at the subpercent level. Therefore, in the presence of the non-local Weylsquare term, scalar perturbations remain well-behaved (at least up to the present time) and in principle consistent with the data.

Conceptually, it is however interesting to observe that, as we extend the time integration more into the future, we start 
to see more and more deviations in the scalar perturbations of the Weyl-square model, compared to $\Lambda \mathrm{CDM}$ or to the pure $R \square^{-2} R$ model. The behavior of the long-wavelength modes can be understood analytically, and we perform the corresponding analysis in Appendix A.

\section{Tensor sector}

We next turn to tensor perturbations, where we will see that the inclusion of the nonlocal Weyl-square term can be more problematic. In the tensor sector we have to solve five coupled second-order differential equations in the variables $h_{i j}, u_{i j}, v_{i j}, \sigma_{i j}, s_{i j}$, coming from the transverse-traceless part of the Einstein equations and of the equations for the auxiliary fields. With the field redefinition (4.22), the relevant equations are

$\hat{s}_{i j}^{\prime \prime}+(\zeta-5) \hat{s}_{i j}^{\prime}-(2+4 \zeta) s_{i j}+\hat{k}^{2} \hat{s}_{i j}=\frac{4}{a h} \hat{\sigma}_{i j}-\frac{1}{h^{2}} u_{i j}$,

$$
\begin{aligned}
\hat{u}_{i j}^{\prime \prime} & +(\zeta-5) \hat{u}_{i j}^{\prime}-(2+4 \zeta) u_{i j}+\hat{k}^{2} u_{i j} \\
& =\frac{4}{a h} \hat{v}_{i j}+\frac{a^{4}}{2}\left(h_{i j}^{\prime \prime}+(1+\zeta) h_{i j}^{\prime}-\hat{k}^{2} h_{i j}\right),
\end{aligned}
$$

$\hat{\sigma}_{i j}^{\prime \prime}+(\zeta-5) \hat{\sigma}_{i j}^{\prime}-(2+4 \zeta) \sigma_{i j}+\hat{k}^{2} \hat{\sigma}_{i j}=-4 a h \hat{k}^{2} \hat{s}_{i j}-\frac{1}{h^{2}} \hat{v}_{i j}$,

$$
\begin{aligned}
\hat{v}_{i j}^{\prime \prime} & +(\zeta-5) \hat{v}_{i j}^{\prime}-(2+4 \zeta) v_{i j}+\hat{k}^{2} \hat{v}_{i j} \\
& =-4 a h \hat{k}^{2} \hat{u}_{i j}+a^{5} \hat{k}^{2} h h_{i j}^{\prime},
\end{aligned}
$$
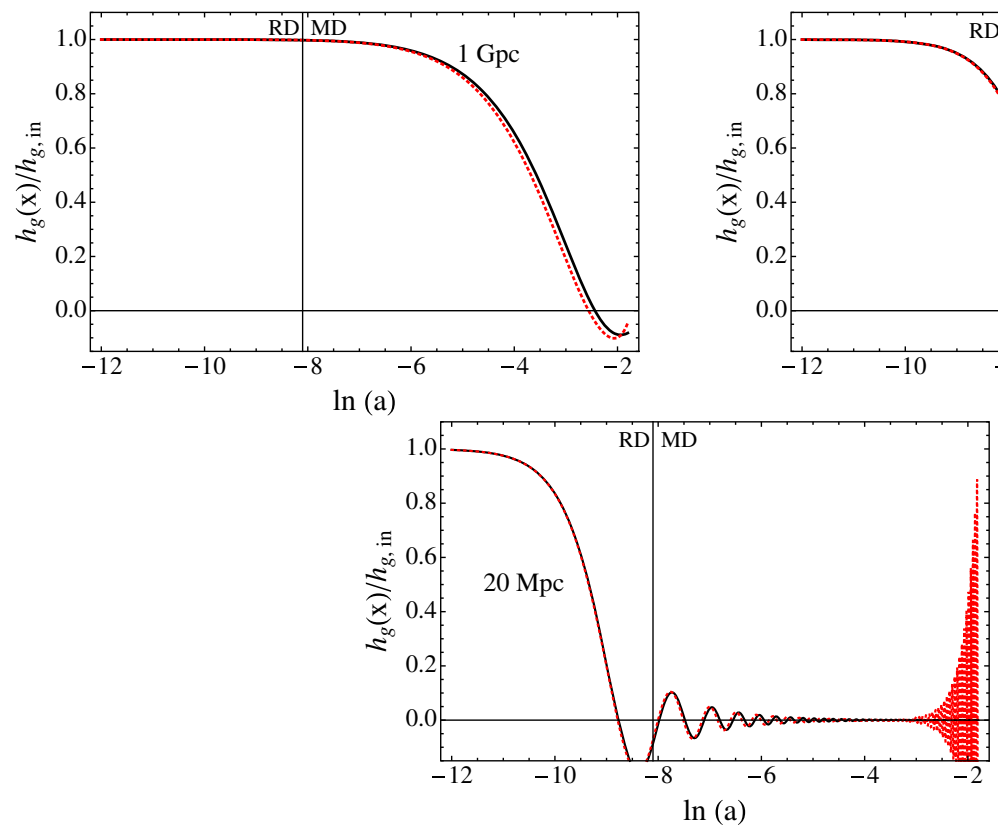

$$
\begin{aligned}
& \left(1-3 \gamma_{1} \bar{V}\right)\left(h_{i j}^{\prime \prime}+(\zeta+3) h_{i j}^{\prime}+\hat{k}^{2} h_{i j}\right)-3 \gamma_{1} \bar{V}^{\prime} h_{i j}^{\prime} \\
& -\frac{6 \gamma_{2}}{a^{4}}\left[\hat{s}_{i j}^{\prime \prime}+(\zeta-3) \hat{s}_{i j}^{\prime}+2(1-\zeta) \hat{s}_{i j}-\hat{k}^{2} \hat{s}_{i j}\right. \\
& \left.+\frac{2}{a h} \hat{\sigma}_{i j}^{\prime}-\frac{4}{a h} \hat{\sigma}_{i j}\right]=0,
\end{aligned}
$$

where in the last equation we have neglected the contribution coming from the anisotropic stress.

As usual, we separate the Fourier components $h_{i j}(\eta, \mathbf{k})$ into their plus and cross polarizations,

$$
h_{i j}(\eta, \mathbf{k})=h_{+}(\eta, \mathbf{k}) e_{i j}^{+}(\mathbf{k})+h_{\times}(\eta, \mathbf{k}) e_{i j}^{\times}(\mathbf{k}),
$$

and similarly for all other transverse-traceless tensors $\hat{s}_{i j}, \hat{u}_{i j}$, etc. The equations for the two polarizations are the same, and we denote generically their amplitudes as $h_{g}$ (not to be confused with the reduced Hubble parameter $\left.h=H / H_{0}\right), s_{g}, u_{g}$, etc.

We solve numerically Eqs. (4.35)-(4.39) starting from an initial redshift $z_{i}=10^{9}$ in radiation domination, with GRlike initial conditions for the metric tensor mode $h_{g}$, i.e. $h_{g}\left(z_{i}\right)=1$ and $h_{g}^{\prime}\left(z_{i}\right)=0$, and vanishing initial conditions for the auxiliary fields $u_{g}, v_{g}, s_{g}$ and $\sigma_{g}$ and their first derivatives. The result of the numerical integration is shown in Fig. 2 where, for clarity, we show separately the result for the same three comoving wavelengths already used in Fig. 1 for scalar perturbations. For each wavelength we now display only the standard $\Lambda \mathrm{CDM}$ result (black solid line) and the result for the Weyl-square model with $\mu_{1}=\mu_{2}=$ $0.014 H_{0}^{2}$ (red, dotted), since the result for the pure $R \square^{-2} R$ model, on this scale, is hardly distinguishable from the

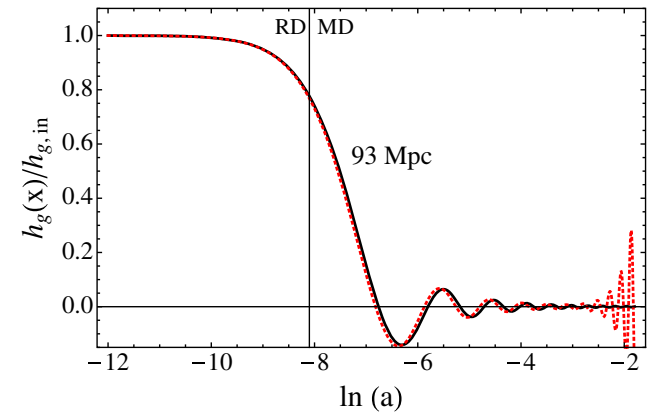

FIG. 2. The evolution of tensor perturbations, for three different comoving reduced wavelengths. For each wavelength we plot the result for $\Lambda \mathrm{CDM}$ (black solid line) and for the model with $\mu_{1}=\mu_{2}=0.014 H_{0}^{2}$ (red, dotted). 
result for $\Lambda \mathrm{CDM}$. We now find the surprise that, in the Weyl-square model, tensor modes become unstable. The instability starts some time after the RD-MD transition and, as we will show analytically in Appendix B, is due to the fact that the auxiliary fields, which are zero during RD, gradually become nonvanishing in MD, and when they are sufficiently large they begin to source the instability. We also observe that the instability is stronger for the short wavelengths, i.e. large frequencies. This is due to the fact that it is actually the time derivative of the auxiliary fields that sources the instability, so the effect is larger for the modes oscillating with large frequency. Observe also that in Fig. 2 we only plot the result up to $\log a=-1.8$. If we extended these plots up to the present time $\log a=0$, the instability would bring the amplitude well outside the vertical scale of the plots. The corresponding plots on a $\operatorname{logarithmic}$ vertical scale, extended up to the $\log a=1$ in the cosmological future, are shown in Fig. 3.

To assess whether this instability in the tensor sector rules out the model which features the nonlocal Weylsquare term, we need to make assumptions on the initial spectrum of perturbation, $h_{\mathrm{g}, \text { in }}(k)$, whose subsequent evolution is then amplified as in Fig. 3. This initial spectrum depends on the theory of the primordial Universe. Some alternatives to the standard slow-roll inflationary paradigm predict a negligible primordial tensor spectrum. For instance, the pre-big-bang model [37] predicts a primordial relic gravitational-wave $(\mathrm{GW})$ spectrum that, in some range of its parameter space, can be sizable, but for other values of the parameters can be totally negligible $[38,39]$. As another example, the ekpyrotic model predicts a negligible amount of primordial tensor perturbations [40,41]. However, to generate an adiabatic spectrum of scalar

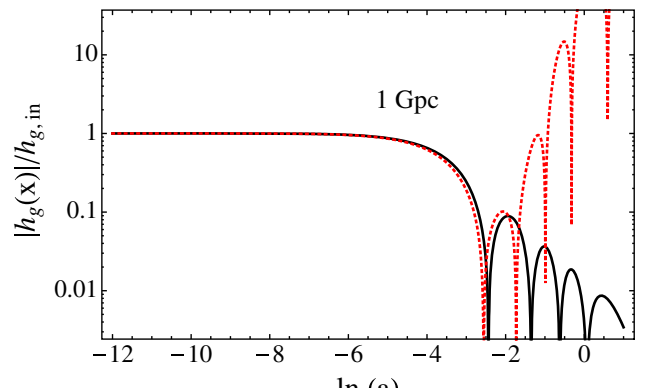

$\ln (\mathrm{a})$ perturbations, the pre-big bang must appeal to mechanisms such as the decay of massive axions, leading to the "curvaton" mechanism [42] which, even if potentially viable, are less natural than the generation of adiabatic perturbations in standard slow-roll inflation.

If we stick to the standard inflationary paradigm, the amplitude of the primordial tensor perturbations is characterized by a tensor-to-scalar ratio $r$, with $r<O(0.1)$ to comply with the joint BICEP2/Keck Array-Planck analysis [43]. Several single-field inflationary models give predictions close to this experimental bound (or in excess of it, in which case they are ruled out). In any case, even the inflationary models that give a small primordial GW spectrum, such as the Starobinsky model, still predict values of $r$ at least of order $10^{-3}$.

In terms of the GW energy density per unit logarithmic interval of frequency, defined as usual as $h_{0}^{2} \Omega_{\mathrm{gw}}(f)=$ $\left(1 / \rho_{c}\right) d \rho_{\mathrm{GW}} / d \log f$ (where the frequency $f$ today is related to $k$ by $k=2 \pi f$ ), the standard functional dependence of the inflationary prediction is $h_{0}^{2} \Omega_{\mathrm{gw}}(f) \propto f^{-2}$ for frequencies $f$ in the range $3 \times 10^{-18} \mathrm{~Hz}<f<f_{\text {eq }}$, where the condition $f>3 \times 10^{-18}$ imposes that the $\mathrm{GW}$ is inside the horizon today, while $f_{\text {eq }} \simeq 1.6 \times 10^{-17} \mathrm{~Hz}$ is the value of the frequency of the mode that reenters the horizon at the RD-MD transitions. Modes with $f>f_{\text {eq }}$ reentered the horizon already during $\mathrm{RD}$, and for these frequencies $h_{0}^{2} \Omega_{\mathrm{gw}}(f) \propto f^{n_{T}}$, where $n_{T}=-r / 8$, so in this regime the spectrum is basically flat (see e.g. Ref. [44] for review).

In the presence of the nonlocal Weyl-square term, this GW spectrum is further amplified by its evolution during $\mathrm{MD}$, as shown in Fig. 3. We can compute this extra amplification by comparing mode by mode the $\Lambda \mathrm{CDM}$
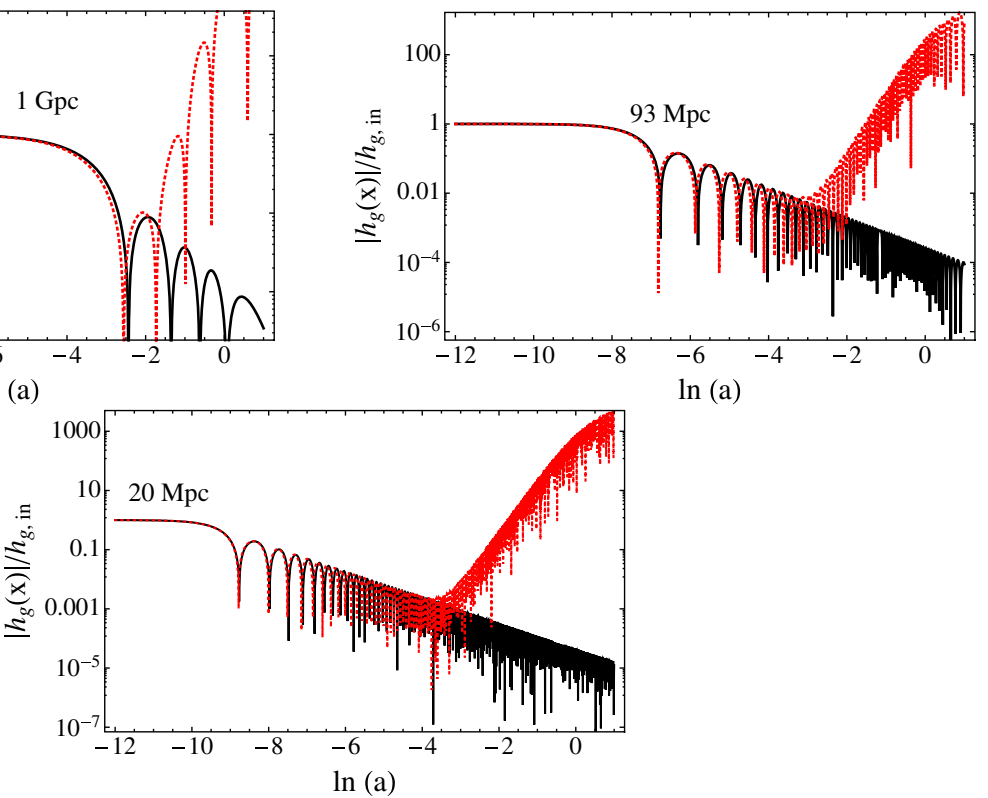

FIG. 3. As in Fig. 2, on a logarithmic vertical scale, and extending the horizontal axis to $\log a=1$. Observe the different vertical scale among the figures. 


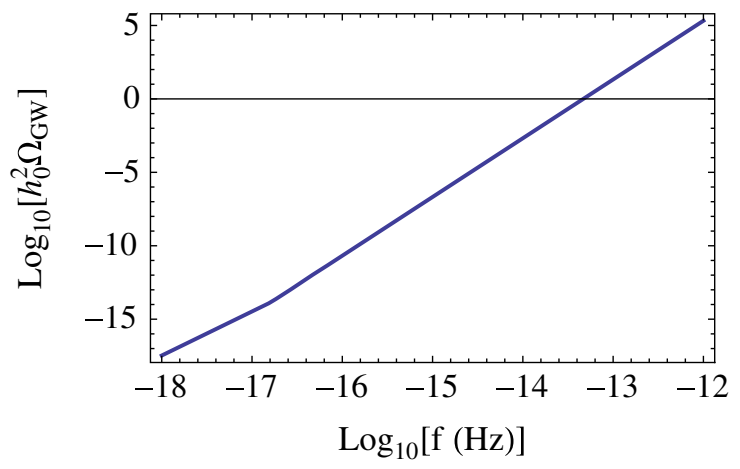

FIG. 4. The value of $h_{0}^{2} \Omega_{\mathrm{gw}}(f)$ for a primordial inflationary spectrum further amplified during the evolution to the present time by the nonlocal Weyl-square term.

result with the result in the presence of the nonlocal Weylsquare term. We find that, because of this further amplification, in the regime $3 \times 10^{-18} \mathrm{~Hz}<f<f_{\text {eq }}$ now $h_{0}^{2} \Omega_{\mathrm{gw}}(f)$ grows as $f^{3}$, while in the regime $f>f_{\mathrm{eq}}$ it grows as $f^{4}$.

Let us denote by $f_{*}=k_{*} /(2 \pi)$ the value of the frequency corresponding to the pivot scale used by Planck. For the typical value $k_{*}=0.002 \mathrm{Mpc}^{-1}$ we have $f_{*} \simeq 3.09 \times 10^{-18} \mathrm{~Hz}$. We denote by $\Omega_{*}$ the corresponding value of $\Omega_{\mathrm{GW}}\left(f=f_{*}\right)$. Then, the form of the inflationary spectrum today, after the further amplification by the Weylsquare term, is

$$
\begin{aligned}
& h_{0}^{2} \Omega_{\mathrm{GW}}(f) \\
& \quad=h_{0}^{2} \Omega_{*} \times \begin{cases}\left(\frac{f}{f_{*}}\right)^{3} & 3 \times 10^{-18} \mathrm{~Hz}<f<f_{\text {eq }}, \\
\left(\frac{f_{\mathrm{eq}}}{f_{*}}\right)^{3}\left(\frac{f}{f_{\mathrm{eq}}}\right)^{4} & f>f_{\text {eq }} .\end{cases}
\end{aligned}
$$

Even assuming a very low value of $h_{0}^{2} \Omega_{*}$, such as $h_{0}^{2} \Omega_{*}=$ $10^{-16}$ (which corresponds to a tensor-to-scalar ratio $r \sim 10^{-4}$ ), such an extremely blue spectrum wildly violates all existing bounds on $h_{0}^{2} \Omega_{\mathrm{gw}}(f)$, coming e.g. from pulsar timing, extra radiation or big bang nucleosynthesis, and even quickly becomes larger than one, as we see from Fig. 4. In principle such a behavior will continue until the intrinsic cutoff of the inflationary spectrum is met, e.g. up to $f=O\left(10^{9}\right) \mathrm{Hz}$ for typical grand-unified-theory-scale inflation. ${ }^{5}$ In this figure we used the result obtained for $\mu_{2}=\mu_{1}=0.014 H_{0}^{2}$ and it is worth recalling that, contrarily

\footnotetext{
${ }^{5}$ Actually, our numerical integration only works up to momenta $k \simeq 10^{4} H_{0}$, i.e. frequencies of order $f \simeq 3.5 \times 10^{-15} \mathrm{~Hz}$. For larger frequencies it becomes numerically difficult to follow reliably the growth of the tensor perturbations. However, such frequencies are already well in the range of frequencies that entered the horizon during RD, so we do not expect a further change of regime until the inflationary cutoff is met.
}

to $\mu_{1}$ which is fixed by the background evolution, $\mu_{2}$ is in principle a free parameter. However it is clear that, unless $\mu_{2}$ is tuned to ridiculously small values, the resulting $\mathrm{GW}$ spectrum is unacceptably large.

The conclusion from this analysis is that, in a cosmological model in which the primordial tensor perturbations are generated by inflation, as well as in any other model in which the primordial tensor perturbations are not completely negligible, the presence of the nonlocal Weyl-square term in the effective action at late time is ruled out by the resulting overproduction of tensor perturbations.

\section{CONCLUSIONS}

The aim of this paper was to study a nonlocal theory of the general form (1.4), which features nonlocal terms proportional to the mass-square parameters $\mu_{1}, \mu_{2}, \mu_{3}$, to see which terms are phenomenologically allowed. The term $\mu_{1} R \square^{-2} R$ has already been studied in a number of papers, and has been shown to produce a viable cosmological model, both at the level of background evolution and of cosmological perturbations. Here we have found that the addition of a term $\mu_{3} R_{\mu \nu} \square^{-2} R^{\mu \nu}$ is quickly ruled out, since it induces instabilities in the background evolution. The case for the nonlocal Weyl-square term, $\mu_{2} C^{\mu \nu \rho \sigma} \square^{-2} C_{\mu \nu \rho \sigma}$, turns out to be more delicate. This term does not contribute to the background evolution. Its scalar perturbations, at least up to the present epoch, are sufficiently close to that of $\Lambda \mathrm{CDM}$ and of the $\mu_{1} R \square^{-2} R$ model, so that they do not pose a threat to the phenomenology of the model (although, for very large wavelengths, they become unstable in the cosmological future). However, the tensor perturbations of the model become unstable already during MD, with the instability being more important for the short wavelengths. This produces a strong amplification of any primordial background of gravitational waves. In particular, if we assume the typical primordial GW background generated by single-field slow-roll inflation, even with very small values of the tensor-to-scalar ratio $r$, the resulting spectrum for $h_{0}^{2} \Omega_{\mathrm{gw}}(f)$ is extremely blue, growing as $f^{4}$ for modes that reentered in $\mathrm{RD}$, leading to a complete violation of all bounds on $h_{0}^{2} \Omega_{\mathrm{gw}}(f)$. Indeed, when increasing the frequency, $h_{0}^{2} \Omega_{\mathrm{gw}}(f)$ even quickly becomes larger than one. Thus, at least in the standard scenario where the initial relic GW background is not totally negligible, the term $\mu_{2} C^{\mu \nu \rho \sigma} \square^{-2} C_{\mu \nu \rho \sigma}$ is also phenomenologically ruled out. ${ }^{6}$

The crucial next step is to understand the mechanism that generates such nonlocalities from a fundamental local theory. The results that we have presented in this paper give a useful hint, since they indicate that we need a mechanism that generates precisely the $R \square^{-2} R$ term [or the

\footnotetext{
${ }^{6} \mathrm{We}$ have found a similar instability in the vector sector of the Weyl-square model, although in this case inflation can dilute to a totally negligible value any initial primordial spectrum.
} 
term in Eq. (1.1)], rather than most general nonlocal structures involving $R_{\mu \nu}$ or the Weyl tensor. A mechanism of this type has been recently suggested in Ref. [6]. The starting point was the observation in Refs. [45,46] that, when gravity is coupled to massless particles, the conformal mode acquires a kinetic term because of the conformal anomaly. In four dimensions the anomalyinduced effective action is

$$
\begin{aligned}
S_{\text {anom }}= & -\frac{1}{8} \int d^{4} x \sqrt{-g}\left(E-\frac{2}{3} \square R\right) \\
& \times \Delta_{4}^{-1}\left[b^{\prime}\left(E-\frac{2}{3} \square R\right)-2 b C^{2}\right],
\end{aligned}
$$

where $E$ is the Gauss-Bonnet combination, $C^{2}$ is the square of the Weyl tensor, $b, b^{\prime}$ are coefficients that depend on the number and type of conformal massless fields, and $\Delta_{4}$ was defined in Eq. (1.7). Restricting the theory to the conformal mode $\sigma$, i.e. writing the metric as $g_{\mu \nu}(x)=e^{2 \sigma(x)}$, the nonlocal action (5.1) becomes local, and reads [45,47]

$$
S_{\text {anom }}=-\frac{Q^{2}}{16 \pi^{2}} \int d^{4} x(\square \sigma)^{2},
$$

where again $Q$ is a coefficient that depends on the number and type of conformal massless fields. The interesting aspect of this result is that, in momentum space, the propagator of the $\sigma$ field goes as $1 / k^{4}$, and therefore has strong IR effects. In particular, the corresponding propagator in coordinate space grows logarithmically, $G\left(x, x^{\prime}\right)=-\left(2 Q^{2}\right)^{-1} \log \left[\mu^{2}\left(x-x^{\prime}\right)^{2}\right]$. The situation is quite similar to what happens in the two-dimensional $\mathrm{XY}$ model. In this case the $1 / k^{2}$ propagator in two dimensions again produces a coordinate-space propagator which grows logarithmically. In the XY model this induces a vortex-vortex interaction that, depending on the value of $Q$, can disorder the system and dynamically generate a mass gap, leading to the well-known Berezinsky-Kosterlitz-Thouless (BKT) transition. It is then quite natural to expect that, in the fourdimensional case, the strong IR effects induced by the $1 / k^{4}$ propagator of the conformal mode might lead to a dynamical mass generation for the conformal mode, similarly to the dynamical mass generation in the BKT transition. In this case, it is precisely the term $m^{2} R \square^{-2} R$ that emerges [6]. Indeed, there is no local diff-invariant term that, when written in terms of the conformal mode and expanded in powers of $\sigma$, gives a mass term for $\sigma$, i.e. starts from a $\sigma^{2}$ term. However, the Ricci scalar computed from the metric $g_{\mu \nu}=e^{2 \sigma(x)} \eta_{\mu \nu}$, to linear order in $\sigma$, is given by $R=-6 \square \sigma+\mathcal{O}\left(\sigma^{2}\right)$. Thus, (upon integration by parts)

$$
m^{2} R \frac{1}{\square^{2}} R=36 m^{2} \sigma^{2}+\mathcal{O}\left(\sigma^{3}\right) .
$$

The nonlocal term $m^{2} R \square^{-2} R$ therefore just provides a diffinvariant way of giving a mass to the conformal mode, while the higher-order interaction terms on the right-hand side of Eq. (5.3) (which are nonlocal even in $\sigma$ ) are required to reconstruct a diff-invariant quantity. The same is true for the model (1.1). Indeed, as we have mentioned, the models (1.1) and (1.2) have the same expansion to linear order over Minkowski space, so the corresponding actions are the same in an expansion to quadratic order over Minkowski space, and they both reproduce the term $\propto \sigma^{2}$ in the action, although with different nonlinear completions.

It is quite interesting to see that the models (1.1) and (1.2) have a physical interpretation, as a mass term for the conformal mode, which is absent for the other nonlocal terms that we have considered, and which could justify the emergence of this specific nonlocal structure. Further work along these lines is of course needed to put this picture on firmer grounds. The results of the present paper however show that, among the possible nonlocal terms of the form (1.4), only the $R \square^{-2} R$ term is phenomenologically acceptable, and this points toward a mechanism, such as the dynamical mass generation for the conformal mode, which would produce exactly this nonlocal structure.

\section{ACKNOWLEDGMENTS}

The work of G. C., S. F. and M. M. is supported by the Fonds National Suisse. The work of M. M. is supported by the SwissMap NCCR.

\section{APPENDIX A: SCALAR PERTURBATIONS WITH $k / H_{0} \ll 1$}

In this appendix we compute analytically the behavior of scalar perturbations with very low values of $k$, e.g. $k / H_{0}=10^{-3}$. As we see from Fig. 1, contrary to what happens in $\Lambda \mathrm{CDM}$, these modes eventually become nonlinear in the cosmological future. This behavior can be understood analytically, as follows.

The evolution of scalar perturbations is governed by Eqs. (4.23)-(4.28) and (4.31)-(4.34). These equations simplify for modes with $k \ll a H$, and the possible onset of an instability can be studied analytically by setting $\gamma_{1}=0$ in the equations for the perturbations (since the perturbations of the $R \square^{-2} R$ model do not show this instability) and working perturbatively in $\gamma_{2}$. Observe that the background evolution is still computed using a nonvanishing $\gamma_{1}$. We consider first the behavior in RD and MD. During radiation and matter dominance, the function $\zeta$ can be approximated as a constant, $\zeta_{0}=\{-2,-3 / 2\}$ respectively. At zeroth order in $\gamma_{2}$ and for $k \ll a H$, Eqs. (4.23)-(4.24) have the GR solution (in both radiation and matter dominance)

$$
\Psi_{0}=-\Phi_{0}=\text { const. }
$$

Plugging this solution into Eq. (4.28), we can solve for the auxiliary field $u$ and the $s$ at zeroth order, and we find that, 
both in $\mathrm{RD}$ and $\mathrm{MD}$, the fastest growing mode is given by the particular solution, $u_{0} \propto a^{2} / h^{2}$ and $s_{0} \propto a^{2} / h^{4}$, respectively. The zeroth-order evolution of the auxiliary fields $u, s$ acts as a source for the first-order correction to the gravitational potentials in Eqs. (4.23)-(4.24), which to this order become

$$
\begin{gathered}
\Phi_{1}^{\prime \prime}+(\zeta+3) \Phi_{1}^{\prime}-\Psi_{1}(3+2 \zeta)-\Psi_{1}^{\prime}=0, \quad(\mathrm{~A} 2) \\
\Psi_{1}=-\Phi_{1}-\frac{6 \gamma_{2}}{a^{2}} h^{2}\left[\hat{s}_{0}^{\prime \prime}+(\zeta-3) \hat{s}_{0}^{\prime}-\left(\frac{5}{4} \zeta-2\right) \hat{s}_{0}+\frac{\hat{k}^{2}}{3} \hat{s}_{0}\right] .
\end{gathered}
$$

The most relevant correction comes from the effective anisotropic stress generated by the effective dark energy in Eq. (4.24), which makes $\Phi \neq \Psi$. The other contributions in Eq. (4.23) are suppressed by extra factors of $\hat{k}^{2}$ and have been neglected in Eq. (A2). Note that we must however retain the term in $\hat{k}^{2}$ in Eq. (4.24) since it may become relevant at late times: indeed, taking for definiteness a MD epoch $\left(h \propto a^{-3 / 2}\right)$, and remembering that $\hat{k}=k /(a h)$, we see that the contribution to the effective anisotropic stress proportional to $k$ on the right-hand side of Eq. (A3) is diluted by a factor $a^{-4}$, while the other source terms are diluted by $h^{2} / a^{2} \propto a^{-5}$. We can solve Eq. (A3) algebraically for $\Psi_{1}$ and use the solution to get a second-order differential equation for $\Phi_{1}$ from Eq. (A2). Then, to first order in $\gamma_{2}$, the particular solution for the gravitational potential $\Phi$ in MD contains a growing mode,

$$
\Phi_{1} \propto k^{2} a / H^{2} \propto a^{4},
$$

which, at late times, dominates over the constant solution. In the left panel of Fig. 5 we show the numerical evolution of the potentials $\Phi$ and $\Psi$ in MD, in the approximation in which the effect of dark energy on the background evolution is neglected (an approximation that, as we know from Sec. IV A, breaks down near the present epoch). In this case, at late times the Universe is dominated by matter and the potentials

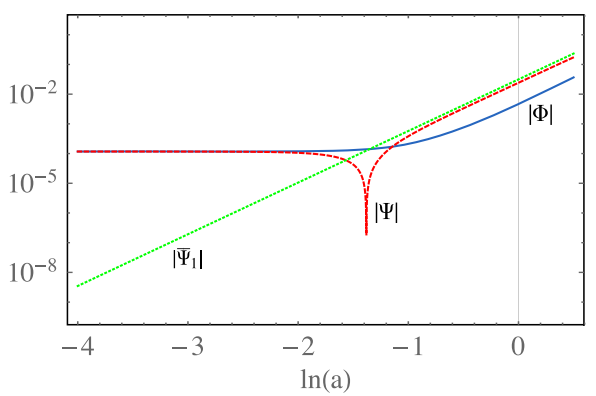

switch from the constant behavior (A1) at the beginning of the MD epoch, to the analytic behavior (A4) (red, dashed line) induced by the growing mode at later times.

We next consider the dark-energy-dominated regime. We mimic it with a de Sitter phase with $\zeta=0$, while $h^{2}$ and $\Omega_{\mathrm{DE}}$ are taken to be constant; we will see that this rather crude approximation (our nonlocal effective dark energy fluid does not behave as a cosmological constant) captures the essential behaviour of superhorizon scales anyway. Then, the growing modes of the auxiliary fields become dominated by the solutions $u \propto a^{\lambda_{+}} / h^{2}, s \propto a^{\lambda_{+}} / h^{4}$, with $\lambda_{+}=(5+\sqrt{33}) / 2 \approx 5.37$. We can then proceed as before and compute the correction to the scalar potentials, which now has a growing mode $\Phi \propto(\alpha+\beta x) a^{\lambda_{+} x} / h^{2}$. In the right panel of Fig. 5, we show the evolution of the potentials on the background given by the full nonlocal model. At late times, the growing mode of the solution in de Sitter becomes dominating. Because of this growing behavior, scalar perturbations of very large wavelength eventually leave the linear regime, even if this happens only in the cosmological future. We see that these analytic estimates allow us to correctly reproduce the qualitative features of the numerical results.

\section{APPENDIX B: ANALYTIC STUDY OF THE INSTABILITIES IN THE TENSOR SECTOR}

In this section we show how to understand analytically some aspects of the evolution of the tensor perturbations, studied numerically in Sec. IV D.

We consider first superhorizon scales. Again, we solve first the equations to zeroth order in $\gamma_{1}$, keeping only the leading growing terms, and we then plug the solution back into the equation for $h_{g}$. To first order in $\gamma_{2}$ we find

$$
\begin{aligned}
h_{g}^{\prime \prime} & +h_{g}^{\prime}+\hat{k}^{2} h_{g} \\
& =\frac{6 \gamma_{2}}{a^{4}}\left[2 s_{g}^{\prime}-2\left(\frac{k}{H_{0}}\right)^{2} \frac{a^{2}}{\Omega_{R}} s_{g}-\frac{a^{4}}{\Omega_{R}} u_{g}-2 a \Omega_{R}^{-1 / 2} \sigma_{g}^{\prime}\right] .
\end{aligned}
$$

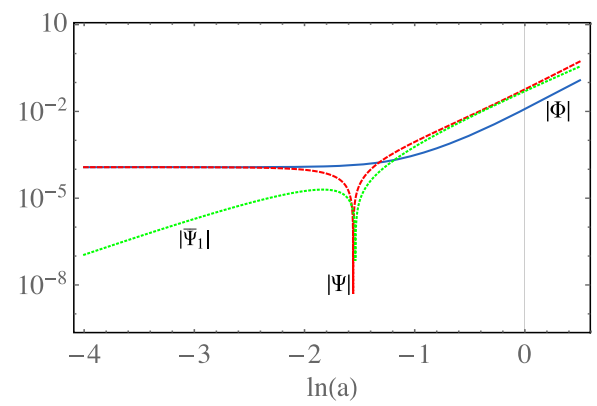

FIG. 5. Numerical evolution of the potentials $\Phi$ (blue, solid line) and $\Psi$ (red, dashed line), with $k=10^{-3} \mathcal{H}_{0}$ and $\gamma_{2}=\gamma_{1}=0.014 H_{0}^{2}$. Left: The background evolution is driven by standard matter and radiation only. The analytical solution for $\Psi$ in matter dominance is the green, dotted line. Right: The background evolution includes dark energy. When the latter becomes relevant at the background level, the solution follows the de Sitter growing mode. 
The tensor mode $h_{q}$ will experience the effects of the source term on the right-hand side of Eq. (B1) when the latter will have grown of order of the $\hat{k}^{2} h_{q}$ term present on the lefthand side. Substituting the solutions for $u_{g}, v_{g} s_{g}$ and $\sigma_{g}$ we find that the leading-order growth of the source term is given by $\sim \gamma_{2}\left(k / H_{0}\right)^{4} \Omega_{R}^{-3} a^{8} \log a$. Therefore, a mode with momentum $k$ will show an instability only for values of the redshift that satisfy $z^{6} \leq \Omega_{R}^{-2} \gamma_{2}\left(k / H_{0}\right)^{2}$. On the other hand, the superhorizon approximation is valid for scales satisfying the condition $z^{6} \gg\left(k / H_{0}\right)^{6} \Omega_{R}^{-3}$. These two conditions cannot be satisfied at the same time, unless an unnaturally large value of $\gamma_{2}$ is chosen. Therefore, modes that enter the horizon after the equality are stable during the entire radiation-dominated epoch. For these modes the effect of the coupling in Eq. (B1) is negligible and their evolution is the standard one in terms of a constant and a decaying mode. This agrees indeed with our numerical result for the mode with $\chi=1 \mathrm{Gpc}$, shown in Fig. 3.

We next consider the late-time instability of subhorizon modes. A full analytic understanding of the growth of subhorizon perturbations in the different epochs is rather complicated since several terms in the solution grow with different powers of the scale factors, but also with different $\hat{\mathbf{k}}$-dependent prefactors. Then, it is quite involved to see, as a function of $\hat{\mathbf{k}}$, what is the term that dominates the growth at any given time. However, it is relatively straightforward to understand the behavior of subhorizon modes at late times, i.e. deep in the DE-dominated epoch (so, in particular, in the cosmological future), since in this case the result is dominated by the terms that grow with the highest power of $a$. As for the scalar case, we approximate the late phase of the evolution of the background as a pure de Sitter phase with $\zeta=0$ and $h=\Omega_{\mathrm{DE}}=$ const. As one can check self-consistently, the fastest-growing modes are the ones associated to the homogeneous solutions of Eqs. (4.35), (4.37), (4.36) and (4.38). These equations have a leading-order growth given by $u_{g}, v_{g}, s_{g}, \sigma_{g} \propto a^{5}$. Making use of this behavior for the auxiliary fields, to

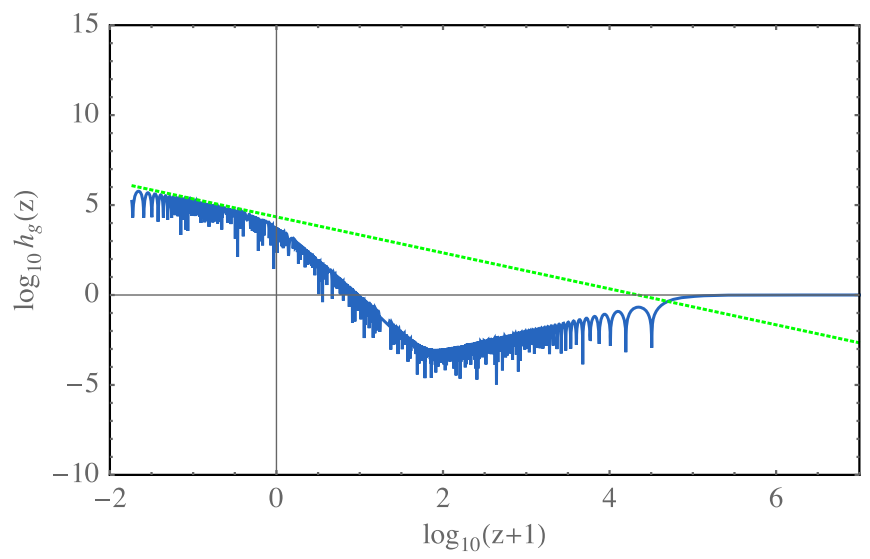

FIG. 6. Numerical evolution of the amplitude of the metric tensor mode $h_{g}$ for $k=10^{3} H_{0}$ and $\gamma_{2}=\gamma_{1}=0.014 H_{0}$. The numerical solution (blue, solid line) is compared to the analytic one valid for subhorizon modes at late times (green, dotted line).

first order in $\gamma_{2}$ the equation for $h_{g}$ can schematically be written as

$h_{g}^{\prime \prime}+4 h_{g}^{\prime}+\hat{k}^{2} h_{g}-\gamma_{2}\left(c_{1} a^{-3}+c_{2} a^{-1}+c_{3} a\right)=0$,

where $c_{1}, c_{2}$ and $c_{3}$ are some coefficients constant in time, but which depend on $\hat{\mathbf{k}}$, as well as on $\Omega_{R}, \Omega_{M}, \Omega_{\mathrm{DE}}$ and $z_{i}$. At late times, the $\mathrm{GW}$ mode $h_{g}$ will then develop a growing mode, with leading-order behavior

$$
h_{g} \propto a .
$$

In Fig. 6 we plot the numerical evolution of a tensor mode with very short wavelength, $k=10^{3} H_{0}$, and we compare it to the slope of the analytic estimate (B3) in the DEdominated epoch [observe that we now plot the result as a function of $\log _{10}(z+1)$, where $z$ is the redshift, so time evolves from right to left]. We see that the analytic estimate correctly reproduces the numerical result at late times.
[1] S. M. Carroll, S. Leichenauer, and J. Pollack, Consistent effective theory of long-wavelength cosmological perturbations, Phys. Rev. D 90, 023518 (2014).

[2] S. Deser and R. Woodard, Nonlocal cosmology, Phys. Rev. Lett. 99, 111301 (2007).

[3] S. Deser and R. Woodard, Observational viability and stability of nonlocal cosmology, J. Cosmol. Astropart. Phys. 11 (2013) 036.

[4] R. Woodard, Nonlocal models of cosmic acceleration, Found. Phys. 44, 213 (2014).

[5] S. Dodelson and S. Park, Nonlocal gravity and structure in the Universe, Phys. Rev. D 90, 043535 (2014).
[6] M. Maggiore, Dark energy and dimensional transmutation in $R^{2}$ gravity, arXiv: 1506.06217 .

[7] N. Arkani-Hamed, S. Dimopoulos, G. Dvali, and G. Gabadadze, Nonlocal modification of gravity and the cosmological constant problem, arXiv:hep-th/ 0209227.

[8] G. Dvali, Predictive power of strong coupling in theories with large distance modified gravity, New J. Phys. 8, 326 (2006)

[9] G. Dvali, S. Hofmann, and J. Khoury, Degravitation of the cosmological constant and graviton width, Phys. Rev. D 76, 084006 (2007). 
[10] M. Porrati, Fully covariant van Dam-Veltman-Zakharov discontinuity, and absence thereof, Phys. Lett. B 534, 209 (2002).

[11] M. Jaccard, M. Maggiore, and E. Mitsou, A non-local theory of massive gravity, Phys. Rev. D 88, 044033 (2013).

[12] M. Maggiore, Phantom dark energy from nonlocal infrared modifications of general relativity, Phys. Rev. D 89, 043008 (2014).

[13] M. Maggiore and M. Mancarella, Non-local gravity and dark energy, Phys. Rev. D 90, 023005 (2014).

[14] S. Foffa, M. Maggiore, and E. Mitsou, Cosmological dynamics and dark energy from non-local infrared modifications of gravity, Int. J. Mod. Phys. A 29, 1450116 (2014).

[15] S. Nesseris and S. Tsujikawa, Cosmological perturbations and observational constraints on nonlocal massive gravity, Phys. Rev. D 90, 024070 (2014).

[16] Y. Dirian, S. Foffa, N. Khosravi, M. Kunz, and M. Maggiore, Cosmological perturbations and structure formation in nonlocal infrared modifications of general relativity, J. Cosmol. Astropart. Phys. 06 (2014) 033.

[17] A. Barreira, B. Li, W. A. Hellwing, C. M. Baugh, and S. Pascoli, Nonlinear structure formation in Nonlocal Gravity, J. Cosmol. Astropart. Phys. 09 (2014) 031.

[18] Y. Dirian, S. Foffa, M. Kunz, M. Maggiore, and V. Pettorino, Non-local gravity and comparison with observational datasets, J. Cosmol. Astropart. Phys. 04 (2015) 044.

[19] Y. Dirian, S. Foffa, M. Kunz, M. Maggiore, and V. Pettorino (in preparation).

[20] L. Modesto and S. Tsujikawa, Non-local massive gravity, Phys. Lett. B 727, 48 (2013).

[21] S. Foffa, M. Maggiore, and E. Mitsou, Apparent ghosts and spurious degrees of freedom in non-local theories, Phys. Lett. B 733, 76 (2014).

[22] A. Kehagias and M. Maggiore, Spherically symmetric static solutions in a non-local infrared modification of general relativity, J. High Energy Phys. 08 (2014) 029.

[23] A. Conroy, T. Koivisto, A. Mazumdar, and A. Teimouri, Generalized quadratic curvature, non-local infrared modifications of gravity and Newtonian potentials, Classical Quantum Gravity 32, 015024 (2015).

[24] G. Cusin, J. Fumagalli, and M. Maggiore, Non-local formulation of ghost-free bigravity theory, J. High Energy Phys. 09 (2014) 181.

[25] Y. Dirian and E. Mitsou, Stability analysis and future singularity of the $m^{2} R \square^{-2} R$ model of non-local gravity, J. Cosmol. Astropart. Phys. 10 (2014) 065.

[26] E. Mitsou, PhD thesis, Geneva University, 2015; Aspects of Infrared Non-local Modifications of General Relativity, arXiv:1504.04050.

[27] A. Barreira, B. Li, E. Jennings, J. Merten, L. King, C. M. Baugh, and S. Pascoli, Galaxy cluster lensing masses in modified lensing potentials, Mon. Not. R. Astron. Soc. 454, 4085 (2015).
[28] A. Barreira, M. Cautun, B. Li, C. Baugh, and S. Pascoli, Weak lensing by voids in modified lensing potentials, J. Cosmol. Astropart. Phys. 08 (2015) 028.

[29] G. Cusin, S. Foffa, M. Maggiore, and M. Mancarella (in preparation).

[30] S. Nojiri and S. D. Odintsov, Modified non-local-F(R) gravity as the key for the inflation and dark energy, Phys. Lett. B 659, 821 (2008).

[31] S. Jhingan, S. Nojiri, S. D. Odintsov, M. Sami, I. Thongkool, and S. Zerbini, Phantom and non-phantom dark energy: The cosmological relevance of non-locally corrected gravity, Phys. Lett. B 663, 424 (2008).

[32] N. Koshelev, Comments on scalar-tensor representation of nonlocally corrected gravity, Gravitation Cosmol. 15, 220 (2009).

[33] T. S. Koivisto, Cosmology of modified (but second order) gravity, AIP Conf. Proc. 1206, 79 (2010).

[34] A. O. Barvinsky, Serendipitous discoveries in nonlocal gravity theory, Phys. Rev. D 85, 104018 (2012).

[35] P. G. Ferreira and A. L. Maroto, A few cosmological implications of tensor nonlocalities, Phys. Rev. D 88, 123502 (2013).

[36] R. Durrer, The Cosmic Microwave Background (Cambridge University Press, Cambridge, England, 2008).

[37] M. Gasperini and G. Veneziano, The pre-big bang scenario in string cosmology, Phys. Rep. 373, 1 (2003).

[38] R. Brustein, M. Gasperini, M. Giovannini, and G. Veneziano, Relic gravitational waves from string cosmology, Phys. Lett. B 361, 45 (1995).

[39] A. Buonanno, M. Maggiore, and C. Ungarelli, Spectrum of relic gravitational waves in string cosmology, Phys. Rev. D 55, 3330 (1997).

[40] J. Khoury, B. A. Ovrut, P. J. Steinhardt, and N. Turok, The ekpyrotic universe: Colliding branes and the origin of the hot big bang, Phys. Rev. D 64, 123522 (2001).

[41] L. A. Boyle, P. J. Steinhardt, and N. Turok, The cosmic gravitational wave background in a cyclic universe, Phys. Rev. D 69, 127302 (2004).

[42] K. Enqvist and M. S. Sloth, Adiabatic CMB perturbations in pre-big bang string cosmology, Nucl. Phys. B626, 395 (2002).

[43] P. Ade et al. (BICEP2/Keck and Planck Collaborations), Joint Analysis of BICEP2/Keck Array and Planck Data, Phys. Rev. Lett. 114, 101301 (2015).

[44] M. Maggiore, Gravitational wave experiments and early universe cosmology, Phys. Rep. 331, 283 (2000).

[45] I. Antoniadis and E. Mottola, 4-D quantum gravity in the conformal sector, Phys. Rev. D 45, 2013 (1992).

[46] I. Antoniadis, P. O. Mazur, and E. Mottola, Criticality and scaling in 4-D quantum gravity, Phys. Lett. B 394, 49 (1997).

[47] I. Antoniadis, P. O. Mazur, and E. Mottola, Cosmological dark energy: Prospects for a dynamical theory, New J. Phys. 9, 11 (2007). 